\title{
Energy dependence of multiplicity fluctuations in heavy ion collisions at $20 \mathrm{~A}$ to $158 \mathrm{~A} \mathrm{GeV}$
}

\author{
C. Alt, ${ }^{9}$ T. Anticic, ${ }^{23}$ B. Baatar, ${ }^{8}$ D. Barna,${ }^{4}$ J. Bartke, ${ }^{6}$ L. Betev,${ }^{10}$ H. Białkowska, ${ }^{20}$ C. Blume, ${ }^{9}$ B. Boimska,${ }^{20}$ M. Botje,${ }^{1}$
}

J. Bracinik, ${ }^{3}$ R. Bramm, ${ }^{9}$ P. Bunčić, ${ }^{10}$ V. Cerny, ${ }^{3}$ P. Christakoglou, ${ }^{2}$ P. Chung,,${ }^{19}$ O. Chvala, ${ }^{14}$ J. G. Cramer, ${ }^{16}$ P. Csató, ${ }^{4}$

P. Dinkelaker, ${ }^{9}$ V. Eckardt, ${ }^{13}$ D. Flierl, ${ }^{9}$ Z. Fodor, ${ }^{4}$ P. Foka, ${ }^{7}$ V. Friese, ${ }^{7}$ J. Gál, ${ }^{4}$ M. Gaździcki,,${ }^{9,11}$ V. Genchev, ${ }^{18}$

G. Georgopoulos, ${ }^{2}$ E. Gładysz,${ }^{6}$ K. Grebieszkow, ${ }^{22}$ S. Hegyi, ${ }^{4}$ C. Höhne, ${ }^{7}$ K. Kadija, ${ }^{23}$ A. Karev, ${ }^{13}$ D. Kikola,${ }^{22}$ M. Kliemant ${ }^{9}$

S. Kniege, ${ }^{9}$ V. I. Kolesnikov, ${ }^{8}$ E. Kornas,${ }^{6}$ R. Korus,${ }^{11}$ M. Kowalski, ${ }^{6}$ I. Kraus, ${ }^{7}$ M. Kreps,${ }^{3}$ A. Laszlo, ${ }^{4}$ R. Lacey, ${ }^{19}$

M. van Leeuwen, ${ }^{1}$ P. Lévai,${ }^{4}$ L. Litov,${ }^{17}$ B. Lungwitz,${ }^{9,}$ M. Makariev, ${ }^{17}$ A. I. Malakhov, ${ }^{8}$ M. Mateev,${ }^{17}$ G. L. Melkumov, ${ }^{8}$

A. Mischke, ${ }^{1}$ M. Mitrovski, ${ }^{9}$ J. Molnár, ${ }^{4}$ St. Mrówczyński, ${ }^{11}$ V. Nicolic,${ }^{23}$ G. Pálla ${ }^{4}$ A. D. Panagiotou, ${ }^{2}$ D. Panayotov, ${ }^{17}$

A. Petridis,${ }^{2, \dagger}$ W. Peryt,${ }^{22}$ M. Pikna,${ }^{3}$ J. Pluta, ${ }^{22}$ D. Prindle, ${ }^{16}$ F. Pühlhofer, ${ }^{12}$ R. Renfordt,${ }^{9}$ C. Roland,${ }^{5}$ G. Roland,${ }^{5}$

M. Rybczyński, ${ }^{11}$ A. Rybicki, ${ }^{6}$ A. Sandoval, ${ }^{7}$ N. Schmitz, ${ }^{13}$ T. Schuster, ${ }^{9}$ P. Seyboth, ${ }^{13}$ F. Siklér, ${ }^{4}$ B. Sitar, ${ }^{3}$ E. Skrzypczak, ${ }^{21}$ M. Slodkowski, ${ }^{22}$ G. Stefanek, ${ }^{11}$ R. Stock,${ }^{9}$ C. Strabel, ${ }^{9}$ H. Ströbele, ${ }^{9}$ T. Susa, ${ }^{23}$ I. Szentpétery, ${ }^{4}$ J. Sziklai, ${ }^{4}$ M. Szuba, ${ }^{22}$ P. Szymanski, ${ }^{10,20}$ V. Trubnikov, ${ }^{20}$ M. Utvic, ${ }^{9}$ D. Varga,,${ }^{4,10}$ M. Vassiliou, ${ }^{2}$ G. I. Veres, ${ }^{4,5}$ G. Vesztergombi, ${ }^{4}$ D. Vranić, A. Wetzler, ${ }^{9}$ Z. Włodarczyk, ${ }^{11}$ A. Wojtaszek, ${ }^{11}$ I. K. Yoo, ${ }^{14}$ and J. Zimányi ${ }^{4, \dagger}$

${ }^{1}$ NIKHEF, Amsterdam, Netherlands

${ }^{2}$ Department of Physics, University of Athens, Athens, Greece

${ }^{3}$ Comenius University, Bratislava, Slovakia

${ }^{4}$ KFKI Research Institute for Particle and Nuclear Physics, Budapest, Hungary

${ }^{5}$ MIT, Cambridge, Massachusetts, USA

${ }^{6}$ Henryk Niewodniczanski Institute of Nuclear Physics, Polish Academy of Sciences, Cracow, Poland

${ }^{7}$ Gesellschaft für Schwerionenforschung (GSI), Darmstadt, Germany

${ }^{8}$ Joint Institute for Nuclear Research, Dubna, Russia

${ }^{9}$ Fachbereich Physik der Universität, Frankfurt, Germany

${ }^{10}$ CERN, Geneva, Switzerland

${ }^{11}$ Institute of Physics Świętokrzyska Academy, Kielce, Poland

${ }^{12}$ Fachbereich Physik der Universität, Marburg, Germany

${ }^{13}$ Max-Planck-Institut für Physik, Munich, Germany

${ }^{14}$ Charles University, Faculty of Mathematics and Physics, Institute of Particle and Nuclear Physics, Prague, Czech Republic

${ }^{15}$ Department of Physics, Pusan National University, Pusan, Republic of Korea

${ }^{16}$ Nuclear Physics Laboratory, University of Washington, Seattle, Washington, USA

${ }^{17}$ Atomic Physics Department, Sofia University St. Kliment Ohridski, Sofia, Bulgaria

${ }^{18}$ Institute for Nuclear Research and Nuclear Energy, Sofia, Bulgaria

${ }^{19}$ Department of Chemistry, SUNY Stony Brook, Stony Brook, New York, USA

${ }^{20}$ Institute for Nuclear Studies, Warsaw, Poland

${ }^{21}$ Institute for Experimental Physics, University of Warsaw, Warsaw, Poland

${ }^{22}$ Faculty of Physics, Warsaw University of Technology, Warsaw, Poland

${ }^{23}$ Rudjer Boskovic Institute, Zagreb, Croatia

(Received 20 December 2007; revised manuscript received 30 July 2008; published 26 September 2008)

\begin{abstract}
Multiplicity fluctuations of positively, negatively, and all charged hadrons in the forward hemisphere were studied in central $\mathrm{Pb}+\mathrm{Pb}$ collisions at $20 \mathrm{~A}, 30 \mathrm{~A}, 40 \mathrm{~A}, 80 \mathrm{~A}$, and $158 \mathrm{~A} \mathrm{GeV}$. The multiplicity distributions and their scaled variances $\omega$ are presented as functions of their dependence on collision energy as well as on rapidity and transverse momentum. The distributions have bell-like shapes and their scaled variances are in the range from 0.8 to 1.2 without any significant structure in their energy dependence. No indication of the critical point in fluctuations are observed. The string-hadronic ultrarelativistic quantum molecular dynamics (UrQMD) model significantly overpredicts the mean, but it approximately reproduces the scaled variance of the multiplicity distributions. The predictions of the statistical hadron-resonance gas model obtained within the grand-canonical and canonical ensembles disagree with the measured scaled variances. The narrower than Poissonian multiplicity fluctuations measured in numerous cases may be explained by the impact of conservation laws on fluctuations in relativistic systems.
\end{abstract}

DOI: 10.1103/PhysRevC.78.034914

PACS number(s): 25.75.Nq, 25.75.Ag

\section{INTRODUCTION}

*Corresponding author: lungwitz@ikf.uni-frankfurt.de

${ }^{\dagger}$ Deceased.
In matter with high energy densities $\left(\approx 1 \mathrm{GeV} / \mathrm{fm}^{3}\right)$, a phase transition is expected between hadrons and a state of quasifree quarks and gluons, the quark-gluon plasma (QGP) 
$[1,2]$. Measurements indicate that this critical energy density is exceeded at the top energies available at the CERN Super Proton Synchrotron (SPS) [3,4] and BNL Relativistic Heavy Ion Collider (RHIC) [5-8] during the early stage of heavy ion collisions. Moreover, the energy dependence of various observables shows anomalies at low SPS energies, which suggest the onset of deconfinement around $30 \mathrm{~A} \mathrm{GeV}$ beam energy in central $\mathrm{Pb}+\mathrm{Pb}$ collisions [9-11].

It was predicted [12] that the onset of deconfinement can lead to a nonmonotonic behavior of multiplicity fluctuations. Lattice QCD calculations suggest furthermore the existence of a critical point in the phase diagram of strongly interacting matter which separates the line of first-order phase transition at high baryo-chemical potentials and low temperature from a crossover at low baryo-chemical potential and high temperature. An increase of multiplicity fluctuations near the critical point of strongly interacting matter is expected [13].

In statistical models, the widths of the multiplicity distributions depend on the conservation laws that the system obeys. Even though for different statistical ensembles the mean multiplicity is the same for sufficiently large volumes, this is not necessarily so for higher moments of the multiplicity distribution, hence multiplicity fluctuations [14]. Fluctuations are largest in the grand-canonical ensemble, where all conservation laws are fulfilled only on average and not on an eventby-event basis. The multiplicity fluctuations are much smaller in the canonical ensemble, where the electric and baryonic charges as well as strangeness are globally conserved. The smallest fluctuations are obtained within the microcanonical ensemble, for which the charges as well as total energy and momentum are conserved. It should be underlined that in nonrelativistic gases, the situation is very different; namely, particle number is conserved in the microcanonical and canonical ensembles, and consequently the total multiplicity in these ensembles does not fluctuate.

These theoretical considerations motivated vigorous theoretical [14-18] and experimental studies of multiplicity fluctuations in high-energy nuclear collisions.

Results on the centrality dependence of multiplicity fluctuations in $\mathrm{Pb}+\mathrm{Pb}$ collisions obtained by the NA49 [19] and WA98 [20] collaborations at top SPS energy show an increase of multiplicity fluctuations with decreasing centrality of the collision in the forward hemisphere. A similar increase of multiplicity fluctuations is observed at midrapidity by the PHENIX [21,22] Collaboration at RHIC energies.

Transverse momentum fluctuations [23] also show a nonmonotonic dependence on system size. They increase from $p+p$ to $\mathrm{Si}+\mathrm{Si}$ and peripheral $\mathrm{Pb}+\mathrm{Pb}$ collisions and decrease from peripheral to central $\mathrm{Pb}+\mathrm{Pb}$ collisions. Possible relations to multiplicity fluctuations are discussed in Refs. [24,25]. Preliminary results of NA49 on the energy dependence of transverse momentum fluctuations [26] in central $\mathrm{Pb}+\mathrm{Pb}$ collisions indicate a constant behavior.

This paper presents the dependence of multiplicity fluctuations on energy as well as on rapidity and transverse momentum for the most central $\mathrm{Pb}+\mathrm{Pb}$ collisions at $20 A, 30 A, 40 A, 80 A$, and $158 A \mathrm{GeV}$ as measured by the NA49 experiment at the CERN SPS.
The paper is organized as follows. In Sec. II, the notation and definitions are presented. In Sec. III, the NA49 experiment and the experimental procedure for selecting events and tracks used for this analysis is described. In Sec. IV, the experimental results on multiplicity fluctuations are shown as a function of energy, rapidity, and transverse momentum [27]. These results are compared with the predictions of the hadron-resonance gas model [16] and the string-hadronic ultrarelativistic quantum molecular dynamics (UrQMD) model [28] in Sec. V. Furthermore, the measurements are also discussed with respect to the search for the onset of deconfinement and the critical point. The paper ends with a summary in Sec. VI.

\section{MEASURE OF MULTIPLICITY FLUCTUATIONS}

Let $P(n)$ denote the probability of observing a particle multiplicity $n\left(\sum_{n} P(n)=1\right)$ in a high-energy nuclear collision.

The scaled variance $\omega$ used in this paper as a measure of multiplicity fluctuations is commonly used in elementary and heavy ion collisions, both for theoretical (see, e.g., Refs. $[16,17,29,30]$ ) and experimental (see, e.g., Refs. [19-21,31]) studies. It is defined as

$$
\omega=\frac{\operatorname{Var}(n)}{\langle n\rangle}=\frac{\left\langle n^{2}\right\rangle-\langle n\rangle^{2}}{\langle n\rangle},
$$

where $\operatorname{Var}(n)=\sum_{n}(n-\langle n\rangle)^{2} P(n)$ and $\langle n\rangle=\sum_{n} n P(n)$ are the variance and mean of the multiplicity distribution, respectively.

In a superposition model, $\omega$ is the same in $A+A$ collisions as in nucleon-nucleon interactions at the same energy per nucleon provided the number of particle-producing sources does not fluctuate from event to event. String-hadronic models predict similar values of $\omega$ for $p+p$ and $\mathrm{Pb}+\mathrm{Pb}$ collisions $[17,30]$. In a hadron-gas model [16], the scaled variance converges quickly to a constant value with increasing volume of the system. In the special case of a hadron-gas model in the grand-canonical formulation [16], neglecting quantum effects and resonance decays, the multiplicity distribution is a Poisson one, namely,

$$
P(n)=\frac{\langle n\rangle^{n}}{n !} e^{-\langle n\rangle} .
$$

The variance of a Poisson distribution is equal to its mean, and thus the scaled variance is $\omega=1$, independent of mean multiplicity.

If there are no particle correlations in momentum space and the single-particle distribution is independent of particle multiplicity, the scaled variance of an arbitrary multiplicity distribution observed in a limited acceptance is related to the scaled variance in the full phase-space (" $4 \pi$ ") as (see Appendix A1 and Refs. $[14,16]$ for derivation):

$$
\omega_{\mathrm{acc}}=\left(\omega_{4 \pi}-1\right) p+1,
$$

where $p$ denotes the fraction of particles measured in the corresponding acceptance. Note that the dependence described by Eq. (3) is violated if effects such as resonance decays, quantum statistics, and energy-momentum conservation introduce correlations in momentum space [32]. 


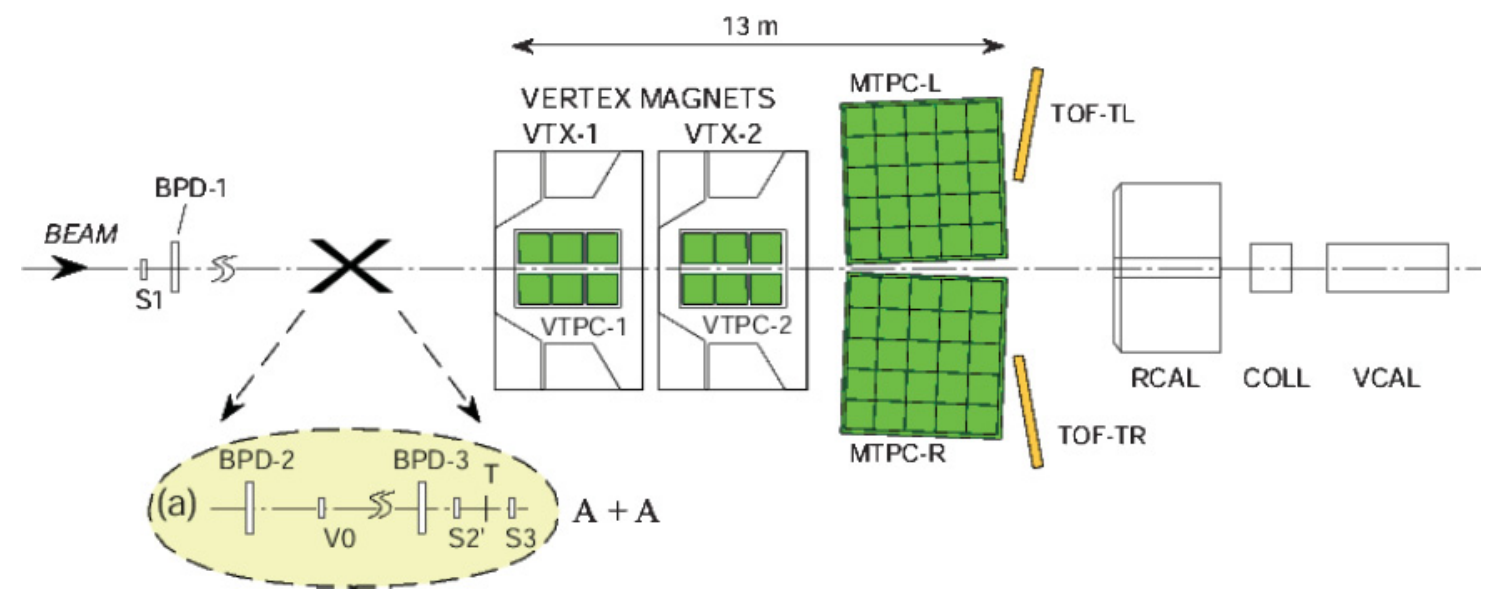

FIG. 1. (Color online) Setup of the NA49 experiment for $\mathrm{Pb}+\mathrm{Pb}$ collisions, see text for more details.

In the following, the scaled variances of the multiplicity distributions of positively, negatively, and all charged hadrons are denoted as $\omega\left(h^{+}\right), \omega\left(h^{-}\right)$, and $\omega\left(h^{ \pm}\right)$, respectively.

\section{THE NA49 EXPERIMENT}

The NA49 detector [33] (see Fig. 1) is a large-acceptance fixed target hadron spectrometer. Its main devices are four large-volume time projection chambers (TPCs). Two of them, called vertex-TPCs (VTPC-1 and -2), are located in two superconducting dipole magnets (VTX-1 and -2) with a total bending power up to $7.8 \mathrm{Tm}$. The magnetic field used at $158 \mathrm{~A} \mathrm{GeV}[\mathrm{B}(\mathrm{VTX}-1) \approx 1.5 \mathrm{~T}$ and $\mathrm{B}(\mathrm{VTX}-2) \approx 1.1 \mathrm{~T}]$ was scaled down in proportion to the beam energy for lower energies. The other two TPCs (MTPC-L and MTPC-R), called main-TPCs, are installed behind the magnets on the left and the right side of the beam line allowing precise particle tracking. The measurement of the energy loss $d E / d x$ in the detector gas provides particle identification in a large momentum range. It is complemented by time-of-flight (TOF) detectors measuring particles at midrapidity. In this analysis, $d E / d x$ information is used only to reject electrons.

The target is located $80 \mathrm{~cm}$ upstream of the first vertex TPC. The target thickness is $0.2 \mathrm{~mm}\left(0.224 \mathrm{~g} / \mathrm{cm}^{2}\right)$ for $20 A-80 A \mathrm{GeV}$ and $0.3 \mathrm{~mm}\left(0.336 \mathrm{~g} / \mathrm{cm}^{2}\right)$ for $158 \mathrm{~A} \mathrm{GeV}$. Using $7.15 \mathrm{~b}$ as the inelastic cross section for $\mathrm{Pb}+\mathrm{Pb}$ collisions, this yields an interaction probability of $0.46 \%$ and $0.7 \%$, respectively. The interaction length of the strong interaction for $\mathrm{Pb}$ ions in a $\mathrm{Pb}$ target is $4.26 \mathrm{~cm}$.

Three beam-position detectors (BPDs) allow a precise determination of the point at which the beam hits the target foil. The centrality of a collision is determined by measuring the energy of projectile spectators in the downstream veto calorimeter (VCAL, see Sec. III B). The acceptance of the veto calorimeter is adjusted at each energy by a proper setup of the collimator (COLL).

\section{A. Data sets and event selection}

This publication presents the results for central $\mathrm{Pb}+\mathrm{Pb}$ collisions at $20 \mathrm{~A}, 30 \mathrm{~A}, 40 \mathrm{~A}, 80 \mathrm{~A}$, and $158 \mathrm{~A} \mathrm{GeV}$. The numbers of events used from these data sets are given in Table I.
To get a "clean" sample of events excluding, for instance, collisions outside the target or event pileup, the following event selection criteria are applied to the data:

(i) The fit of the interaction point, based on the reconstructed tracks, was successful.

(ii) The position of the fitted interaction point is close to the position obtained from the beam position detectors.

(iii) At least $10 \%$ of all tracks are used for the reconstruction of the interaction point. The reconstruction of the interaction point was optimized for precision by selecting long and well-measured tracks in an iterative procedure.

The event cuts have a small influence on $\omega$; the results differ by less than $1 \%$ when only the cut requirement of a successful fit of the main vertex is used.

Beam lead ions that do not interact strongly in the target produce delta electrons both in the target foil and the detector gas. These electrons might curl up in the TPCs, increase their occupancy, and thereby reduce the reconstruction efficiency. To avoid this effect, only those events are selected for the analysis in which there are no beam ions passing through the detector within the readout time of the event.

\section{B. Centrality selection}

Fluctuations in the number of participants lead to an increase of multiplicity fluctuations. In a superposition model, the total multiplicity $n$ is the sum of the number of particles

TABLE I. Statistics for the $1 \%$ most central collisions used for this analysis at different beam energies.

\begin{tabular}{cc}
\hline \hline Energy $(\mathrm{GeV})$ & No. of events \\
\hline $20 A$ & 6602 \\
$30 A$ & 8219 \\
$40 A$ & 21995 \\
$80 A$ & 2307 \\
$158 A$ & 5493 \\
\hline \hline
\end{tabular}


produced by $k$ particle-production sources:

$$
n=\sum_{i} n_{i}^{\text {so }},
$$

where the summation index $i$ runs over the sources. Under the assumption of statistically identical sources, the scaled variance $\omega$ of the multiplicity distribution has two contributions. The first is due to the fluctuations of the number of particles emitted by a single source $\omega_{\text {so }}$, the second is due to the fluctuations in the number of sources $\omega_{k}$ (see Appendix A2 for derivation):

$$
\omega=\omega^{\mathrm{so}}+\left\langle n^{\mathrm{so}}\right\rangle \omega_{k},
$$

where $\left\langle n^{\text {so }}\right\rangle$ is the mean multiplicity of hadrons from a single source. The fluctuations in the number of sources $\omega_{k}$ can be attributed to fluctuations in the number of projectile and target participants. To minimize the fluctuations of the number of participants, the centrality variation in the ensemble of events should be as small as possible, for which very central collisions are best suited.

To fix the number of projectile participants, the NA49 experiment uses the energy in the projectile spectator domain as a measure of centrality, called "projectile centrality" below. The downstream veto calorimeter [34] of NA49, originally designed for NA5, measures the energy carried by the particles in the projectile spectator phase-space region [35]. A collimator in front of the calorimeter is located $25 \mathrm{~m}$ downstream from the target and is adjusted for each energy in such a way that all projectile spectator protons, neutrons, and fragments can reach the veto calorimeter. For $158 \mathrm{~A} \mathrm{GeV}$, the hole in the collimator extends $\pm 5 \mathrm{~cm}$ in the vertical direction and $-5 \mathrm{~cm}$ and $+38 \mathrm{~cm}$ in the horizontal direction taking into account the deflection of charged spectators by the magnetic field (Fig. 2, Table II). Because of a larger spread of spectators, the hole of the collimator is larger for $40 A$ and $80 A \mathrm{GeV}$. For $20 \mathrm{~A}$ and $30 \mathrm{~A}$, the collimator is removed, and the ring calorimeter (RCAL in Fig. 1) positioned $18 \mathrm{~m}$ downstream from the target serves as a collimator.

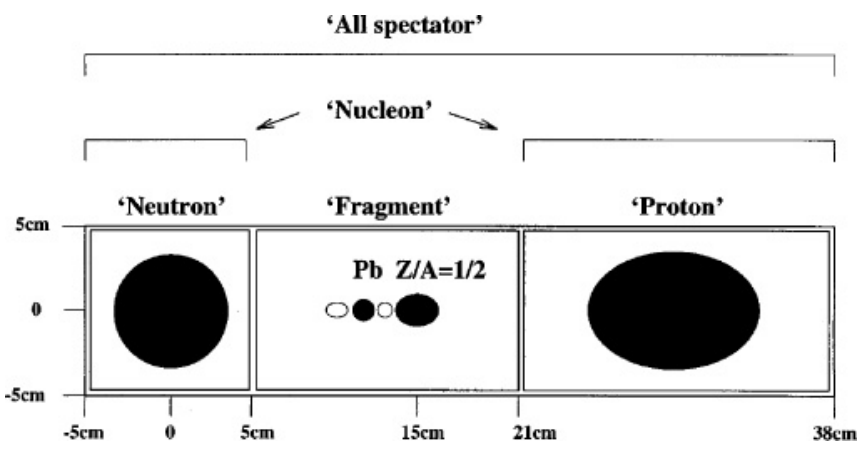

FIG. 2. A sketch of the horizontal deflection for charged particles at the front face of the iron collimator for the $158 \mathrm{~A} \mathrm{GeV}$ magnetic field setting. The broadened distribution of each species is due to the Fermi motion of nucleons or fragments; additionally, the oval shapes are due to the deflection of charged particles in the magnetic field. The sizes of the distributions correspond to one standard deviation. The open circles in the fragment acceptance represent particles of $Z / A$ other than one-half [35].
TABLE II. Settings of the collimator and ring calorimeter defining the acceptance of the veto calorimeter for different energies with respect to the position of neutrons with zero transverse momentum.

\begin{tabular}{lccc}
\hline \hline \multirow{2}{*}{ Energy $(\mathrm{GeV})$} & \multicolumn{2}{c}{ Collimator } & \multirow{2}{*}{ Ring cal. $x(\mathrm{~cm})$} \\
\cline { 2 - 3 } & $x(\mathrm{~cm})$ & $y(\mathrm{~cm})$ & \\
\hline $20 A$ & & & 10 \\
$30 A$ & & & 10 \\
$40 A$ & $-13+47$ & \pm 12 & 17 \\
$80 A$ & $-13+47$ & \pm 12 & 17 \\
$158 A$ & $-7+38$ & \pm 5 & 17 \\
\hline \hline
\end{tabular}

The settings of the hole in the collimator and the position of the ring calorimeter for the different energies is shown in Table II. The zero point is the point at which neutrons with no transverse momentum would pass the collimator. The collimator is not symmetric around the zero point, because the nuclear fragments and spectator protons carry a positive charge and are deflected by the magnetic field in the positive $x$ direction. The last column in the table is the position of the center of the ring calorimeter. Its hole has a radius of $28 \mathrm{~cm}$.

The acceptance of the veto calorimeter for neutral and positive particles for $158 \mathrm{~A} \mathrm{GeV}$ is shown in Fig. 3. Acceptance tables in $p, p_{T}$, and $\phi$ can be obtained at Ref. [36].

Because of the geometry of the collimator and the magnetic field, a small number of positive and neutral nonspectator particles can hit the veto calorimeter. For positively charged
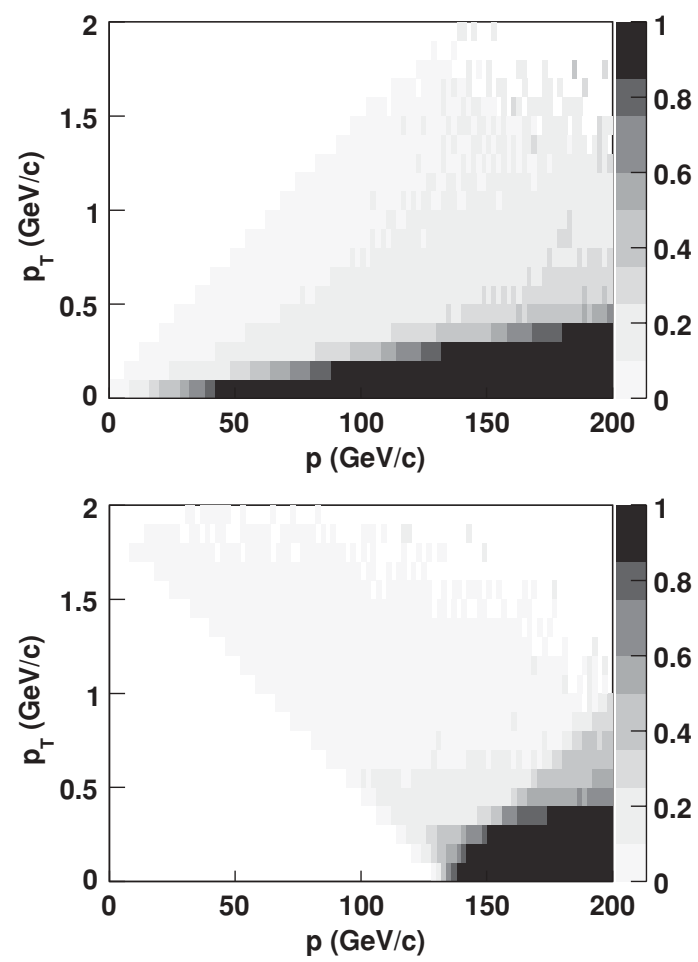

FIG. 3. Acceptance of the veto calorimeter for neutral (top) and positively charged (bottom) main vertex particles at $158 \mathrm{~A} \mathrm{GeV}$ as a function of total momentum $p$ and transverse momentum $p_{T}$. 
particles, the acceptance of the TPCs and the veto calorimeter partly overlap. The maximum amount of a possible autocorrelation is estimated by a comparison of $\omega\left(h^{+}\right)$for UrQMD events selected by their veto energy to UrQMD events with a zero impact parameter in the forward region (Fig. 21) and found to be smaller than $3 \%$.

The acceptance of the veto calorimeter for negatively charged particles is very small, because they are bent by the magnetic field into the direction opposite to the one of the positively charged particles, and the collimator is adjusted to detect positively charged and neutral projectile spectators.

The projectile centrality $C_{\text {Proj }}$ of an event with a veto energy $E_{\text {Veto }}$ is defined as the percentage of all inelastic events that are as central as or more central than the given event according to the energy deposited in the veto calorimeter by the projectile spectator nucleons. Smaller $C_{\text {Proj }}$ correspond to more central events. Using the fraction of inelastic cross section $C_{\text {trig }}=\frac{\sigma_{\text {trig }}}{\sigma_{\text {inel }}}$ accepted by the trigger ( $\sigma_{\text {trig }}$ is derived from the target thickness and the interaction rate; $\sigma_{\text {inel }}$ is assumed to be $7.15 \mathrm{~b}$ ) and the veto energy distribution $C_{\text {Proj }}$ is given by

$$
C_{\text {Proj }}=C_{\text {trig }} \frac{\int_{0}^{E_{\text {Veto }}} d N / d E_{\text {Veto,trig }} d E_{\text {Veto }}}{\int_{0}^{\infty} d N / d E_{\text {Veto,trig }} d E_{\text {Veto }}},
$$

where $d N / d E_{\text {Veto,trig }}$ is the veto calorimeter energy distribution for a given trigger.

The finite resolution of the veto calorimeter causes additional fluctuations in the number of participants. Based on the analysis of the $\mathrm{NA} 49 \mathrm{~Pb}+\mathrm{Pb}$ data, the resolution of the veto calorimeter was estimated in Ref. [19] to be

$$
\frac{\sigma\left(E_{\mathrm{Veto}}\right)}{E_{\mathrm{Veto}}} \approx \frac{2.85}{\sqrt{E_{\mathrm{Veto}}}}+\frac{16}{E_{\mathrm{Veto}}},
$$

where $E_{\text {Veto }}$ is in units of $\mathrm{GeV}$. To check this parametrization, the distribution of the spectators was simulated by the SHIELD model [37]. The SHIELD model delivers both spectator nucleons and nuclear fragments, in contrast to most stringhadronic models, which only produce spectator nucleons. A simulation performed at $20 \mathrm{~A}$ and $158 \mathrm{~A} \mathrm{GeV}$ including the geometry of the NA49 detector and the nonuniformity of the veto calorimeter confirms the parametrization given by Eq. (7) as an upper limit (see Fig. 4).

The veto calorimeter response can in principle change with time (aging effects, etc.). Therefore a time-dependent calibration of the veto energy was applied. The contribution of this correction to $\omega$ turned out to be very small $(<1 \%$, see Table IV).

When fixing the projectile centrality $C_{\text {Proj }}$ [Eq. (6)], thereby fixing the number of projectile participants $N_{P}^{\text {Proj }}$, the number of target participants $N_{P}^{\text {targ }}$ can still fluctuate. Thus the total number of participants is not rigorously constant and could contribute to fluctuations. The fluctuations of the number of target participants obtained by UrQMD and hadron-string dynamics (HSD) simulations [38], expressed as their scaled variance $\omega_{P}^{\text {targ }}=\operatorname{Var}\left(N_{P}^{\text {targ }}\right) /\left\langle N_{P}^{\text {targ }}\right\rangle$, are shown in Fig. 5 . For noncentral collisions, the number of target participants strongly fluctuates, even for a fixed number of projectile participants. This is consistent with the increase of $\omega$ with
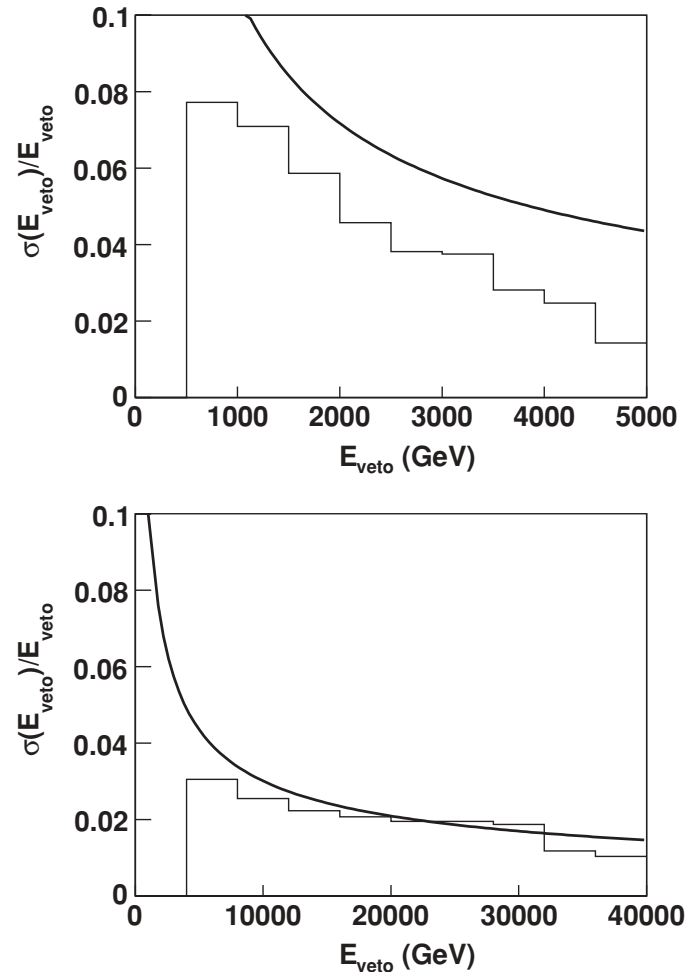

FIG. 4. Resolution of the veto calorimeter estimated by a SHIELD simulation (histogram) compared to the parametrization Eq. (7) (solid line) for $20 \mathrm{~A}$ (top) and 158 A GeV (bottom).

decreasing centrality observed in the forward hemisphere [19,39]. However, alternative explanations also exist [25,40].

For further analysis, the $1 \%$ most central collisions (according to their veto energy) are selected in order to minimize the fluctuations in the number of participants. For these very central collisions, the fluctuation in the number of target participants is expected to be smallest and its scaled variance $\omega_{P}^{\text {targ }}$ is expected to be about 0.1 (see Fig. 5) for an estimated number of target participants of $N_{P}^{\text {targ }} \approx 192$.

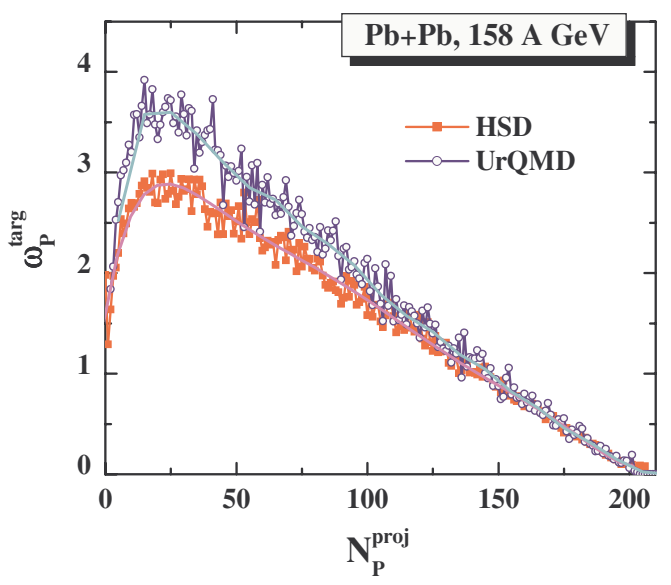

FIG. 5. (Color online) Scaled variance of the number of target participants for a fixed number of projectile participants in the UrQMD and HSD models. The plot is taken from Ref. [38]. 
To estimate the effect on $\omega$ of target participant fluctuations and nonspectator particles in the veto calorimeter, the energy dependence of the scaled variance of the multiplicity distribution is calculated in the UrQMD 1.3 model both for collisions with zero impact parameter and for collisions selected according to their veto energy. The resulting difference of $\omega$ in the forward acceptance (see Sec. III C) is smaller than $2 \%$ for negatively, smaller than $3 \%$ for positively, and smaller than $4 \%$ for all charged hadrons. In the midrapidity region, the influence of the fluctuations of target participants on $\omega$ is expected to be much larger. Indeed, the differences of $\omega$ increase to up to $6 \%$ for negative, up to $9 \%$ for positive, and up to $13 \%$ for all charged hadrons.

To check the influence of the centrality selection, $\omega$ was also determined for the $0.5 \%$ most central collisions. The change from the values obtained for the $1 \%$ most central collisions is less than $3 \%$ for positive, $2 \%$ for negative, and $5 \%$ for all charged hadrons.

\section{Track selection}

Since detector effects such as track reconstruction efficiency might have a significant influence on multiplicity fluctuations, it is important to select a sample of well-defined tracks for the analysis. The following track selection criteria are used for this analysis and are explained in this section:

(i) Number of potential points (the number of points a track can have according to its geometry) in the TPCs: $>30$.

(ii) The ratio of the number of reconstructed points to the number of potential points: $>0.5$.

(iii) Sum of the number of reconstructed points in VTPC-1 and $-2:>5$.

(iv) Sum of the number of reconstructed points in VTPC-2 and MTPCs: $>5$.

(v) The track is extrapolated to the plane of the target foil. This point must be closer than $4 \mathrm{~cm}$ in $x$ and $2 \mathrm{~cm}$ in $y$ direction to the interaction point of the collision.

(vi) To exclude electrons from the analysis, a cut on the energy loss $(d E / d x)$ in the detector gas was applied. All tracks with an energy loss of more than 0.2 minimum ionizing units higher than the pion $d E / d x$ (in the region of the relativistic rise of the Bethe-Bloch formula) are rejected.

The reconstruction efficiency is calculated using the embedding method. Events containing a few tracks were generated and processed by the simulation software. The resulting raw data were embedded into real events. The combined raw data were reconstructed, and the input tracks were matched with the reconstructed ones. Embedding simulations show a significant decrease of reconstruction efficiency with increasing event multiplicity in the midrapidity region at $158 \mathrm{~A} \mathrm{GeV}$ using the track selection criteria described above. Therefore for this energy, an additional cut was used, namely, that tracks should have at least five reconstructed points both in VTPC-2 and in the MTPCs. For these tracks, no significant dependence of reconstruction efficiency on track multiplicity is observed.
TABLE III. Fraction (in percent) of negatively charged main vertex pions, kaons, and antiprotons in different rapidity intervals for different collision energies which are accepted and reconstructed. In addition, the width of the rapidity distribution of negatively charged pions is given $[9,10]$.

\begin{tabular}{lcccc}
\hline \hline $\begin{array}{l}\text { Energy } \\
(\mathrm{GeV})\end{array}$ & $\begin{array}{c}0<y(\pi) \\
<y_{\text {beam }}\end{array}$ & $\begin{array}{c}0<y(\pi) \\
<1\end{array}$ & $\begin{array}{c}1<y(\pi) \\
<y_{\text {beam }}\end{array}$ & $\sigma(y)\left(\pi^{-}\right)$ \\
\hline $20 \mathrm{~A}$ & $15.3 \%$ & $7.2 \%$ & $8.1 \%$ & 1.01 \\
$30 \mathrm{~A}$ & $19.1 \%$ & $8.4 \%$ & $10.7 \%$ & 1.08 \\
$40 \mathrm{~A}$ & $21.7 \%$ & $9.2 \%$ & $12.6 \%$ & 1.1 \\
$80 \mathrm{~A}$ & $28.2 \%$ & $11.2 \%$ & $17 \%$ & 1.23 \\
$158 \mathrm{~A}$ & $28.8 \%$ & $9.6 \%$ & $19.2 \%$ & 1.38 \\
\hline \hline
\end{tabular}

Reconstruction inefficiencies mostly occur for tracks with a very low number of points in the TPCs or for tracks that only have points in the VTPC-1 or in the main TPC. These tracks are not used for this analysis.

In the following, the longitudinal motion of particles is characterized by the rapidity in the center-of-mass system assuming pion mass of the particle. This measure is called pion rapidity and is denoted as $y(\pi)$.

The distributions of the registered tracks after applying the track selection criteria are shown in Fig. 6 as a function of pion rapidity $y(\pi)$ and transverse momentum $p_{T}$. Acceptance tables in $y(\pi), p_{T}$, and $\phi$ can be obtained from Ref. [36]. Only tracks in the rapidity interval starting at midrapidity and ending at beam rapidity are used.

To study the multiplicity fluctuations differentially, the pion rapidity interval $0<y(\pi)<y_{\text {beam }}$ is divided into two parts: the midrapidity $[0<y(\pi)<1]$ and the forward rapidity $\left[1<y(\pi)<y_{\text {beam }}\right]$ regions (see Fig. 7). The fractions of total charged particle multiplicity falling into the different rapidity intervals are given in Table III and Fig. 8. The values are calculated using the VENUS event generator [41] as input for a GEANT-based simulation. The tracks produced by GEANT are converted into detector signals and reconstructed by the NA49 reconstruction chain. For the determination of the acceptance, the negatively charged main vertex pions, kaons, and antiprotons are used. In both regions, a similar number of particles are detected by NA49. In the forward acceptance, the particles are mostly passing through both the vertex and the main TPCs and are therefore efficiently reconstructed for all collision energies. According to the UrQMD model, the fluctuations in the number of target participants contribute mostly to the particle number fluctuations in the target hemisphere and the midrapidity region. Their influence on $\omega$ in the forward region $[y(\pi)>1]$ can be estimated by the difference in scaled variance between $b=0$ and veto selected collisions (see Sec. III B) and is about $1-2 \%$.

Note that the acceptance used for this analysis is larger than the one used for the preliminary data shown in Refs. [42,43].

\section{Systematic errors}

The influence of the selection criteria described above on the scaled variance $\omega$ of the multiplicity distribution has 

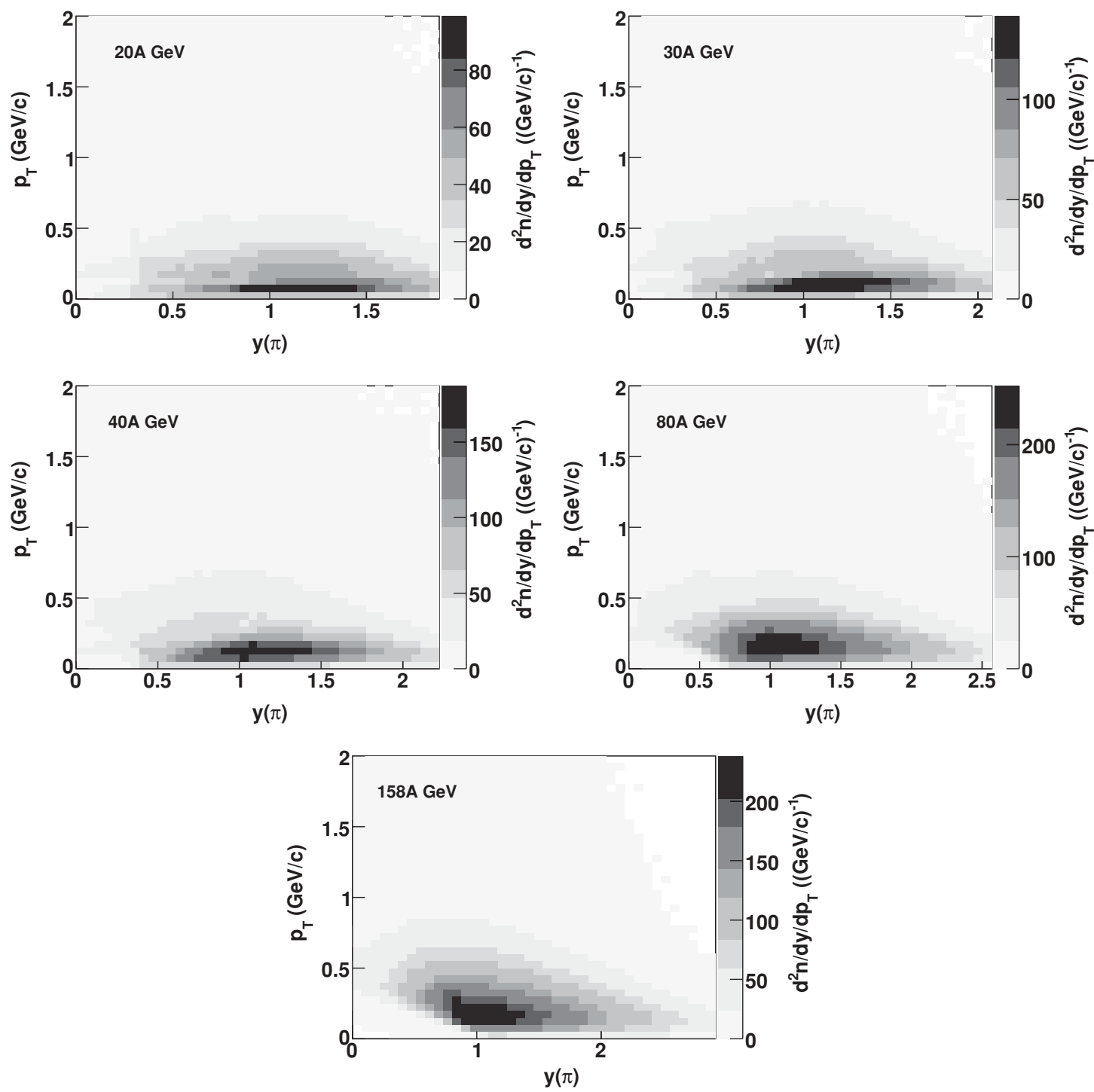

FIG. 6. Distribution of detected negatively charged particles that fulfill the track selection criteria as a function of $y(\pi)$ and $p_{T}$ for $20 A, 30 A, 40 A, 80 A$, and $158 A \mathrm{GeV}$.

been studied, and the results are presented in Table IV and Figs. 9-11. The event selection criteria described in Sec. III A change $\omega$ by up to $2 \%$ from the value obtained when not applying these cuts. The finite resolution of the veto calorimeter causes additional fluctuations in the number of projectile participants and therefore increases the measured $\omega$. In a superposition model, the effect of the veto calorimeter resolution is estimated to be [19]

$$
\delta=\frac{\langle N\rangle \operatorname{Var}\left(E_{\mathrm{Veto}}\right)}{\left(E_{\text {beam }} N_{P}^{\text {Proj }}\right)^{2}},
$$

where $E_{\text {beam }}$ is the total energy per projectile nucleon. The parametrization in Eq. (7), which serves as an upper limit of the resolution of the calorimeter, was used to determine the potential influence of the resolution on $\omega$. For the very central collisions selected for this analysis, the measured $\omega$ is found to increase due to the finite calorimeter resolution by less than $1.5 \%$. Therefore a correction for this effect is not applied. To take possible aging effects of the calorimeter (see Sec. III B) into account, a time-dependent calibration is applied to the measured veto energy. However, the effect of this correction is very small: $\omega$ changes by less than $1 \%$. Track selection criteria are applied to remove electrons and tracks not originating from the main interaction point. The value of $\omega$ is changed by less than $1.5 \%$ for positively and negatively and less than $3 \%$ for all charged hadrons when removing these cuts.

Embedding simulations demonstrated that the reconstruction efficiency shows no significant decrease with increasing particle multiplicity. Therefore no systematic error due to reconstruction efficiency was attributed. The overall reconstruction efficiency is about $95 \%$ and is included in the calculation of the acceptances (Fig. 8, Table III).

The total systematic error is calculated by adding the contributions of the different error sources in quadrature. It is $2.4 \%, 1.8 \%$, and $3.8 \%$ for positively, negatively, and all charged hadrons, respectively. 
TABLE IV. Maximum change $\Delta \omega$ of the scaled variance $\omega$ of the multiplicity distribution for positively, negatively, and all charged hadrons when applying a correction or neglecting a cut. The systematic errors are calculated by adding the error contributions in quadrature. The last row shows the change of $\omega$ resulting from a change in the centrality selection from $1 \%$ to $0.5 \%$.

\begin{tabular}{lccc}
\hline \hline & $\Delta \omega^{+}(\%)$ & $\Delta \omega^{-}(\%)$ & $\Delta \omega^{ \pm}(\%)$ \\
\hline $\begin{array}{l}\text { Event selection } \\
\text { Calorimeter }\end{array}$ & 1.5 & 1 & 1.5 \\
$\begin{array}{l}\text { resolution } \\
\text { Calorimeter }\end{array}$ & 1 & 0.5 & 1.5 \\
$\begin{array}{l}\text { calibration } \\
\text { Track selection }\end{array}$ & 0.5 & 1 & 1 \\
$\begin{array}{l}\text { Total systematic } \\
\text { error }\end{array}$ & 2.4 & 1.5 & 3 \\
$\begin{array}{l}0.5 \% \text { vs } 1 \% \text { most } \\
\text { central }\end{array}$ & 3 & 1.8 & 3.8 \\
\hline
\end{tabular}

To estimate the effect of centrality selection, the $0.5 \%$ most central collisions are also studied. The result for $\omega$ for this stricter selection is up to $5 \%$ different from that obtained for the $1 \%$ most central collisions. As the centrality selection is a welldefined procedure and can be repeated in model calculations,
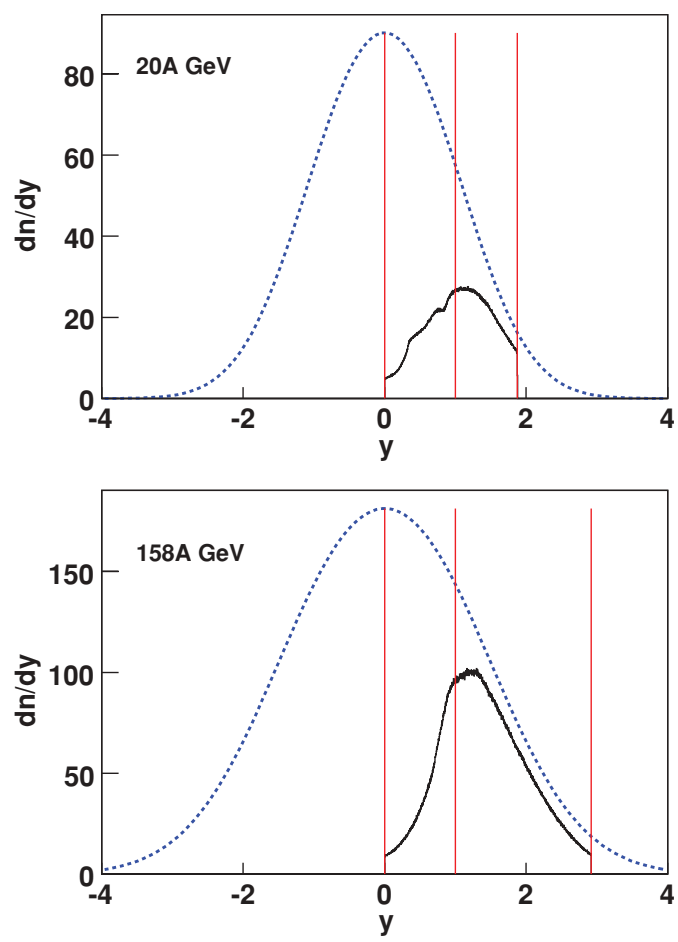

FIG. 7. (Color online) Dashed line shows the double-Gauss parametrization of the rapidity distribution of negatively charged pions and kaons in $\mathrm{Pb}+\mathrm{Pb}$ collisions at $20 A$ [10] and $158 \mathrm{~A} \mathrm{GeV}$ [9]. Solid line is the measured $y(\pi)$ distribution with the track selection criteria described in Sec. III C. The vertical lines indicate the limits of the rapidity intervals $y(\pi)=0, y(\pi)=1$, and $y(\pi)=y_{\text {beam }}$ used for this analysis.

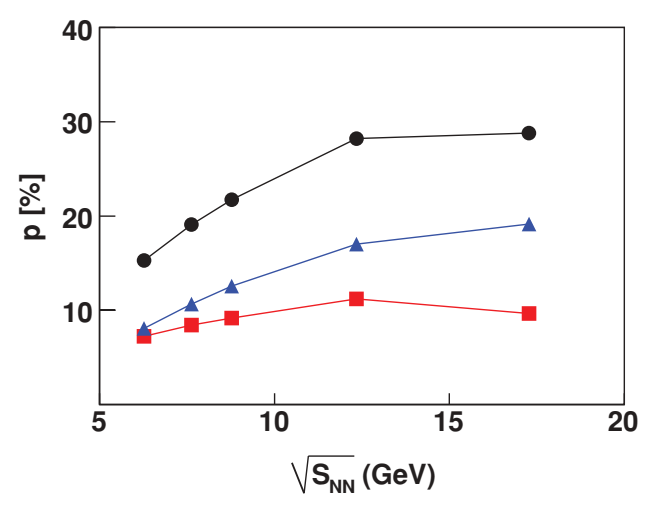

FIG. 8. (Color online) Fraction $p$ of total negatively charged main vertex pion, kaon, and antiproton multiplicity which is accepted and reconstructed as a function of collision energy. Circles: $0<y(\pi)<$ $y_{\text {beam }}$; boxes: $0<y(\pi)<1$; triangles: $1<y(\pi)<y_{\text {beam }}$.

the difference of $\omega$ for the $0.5 \%$ and $1 \%$ most central collisions is not considered as part of the systematic error.
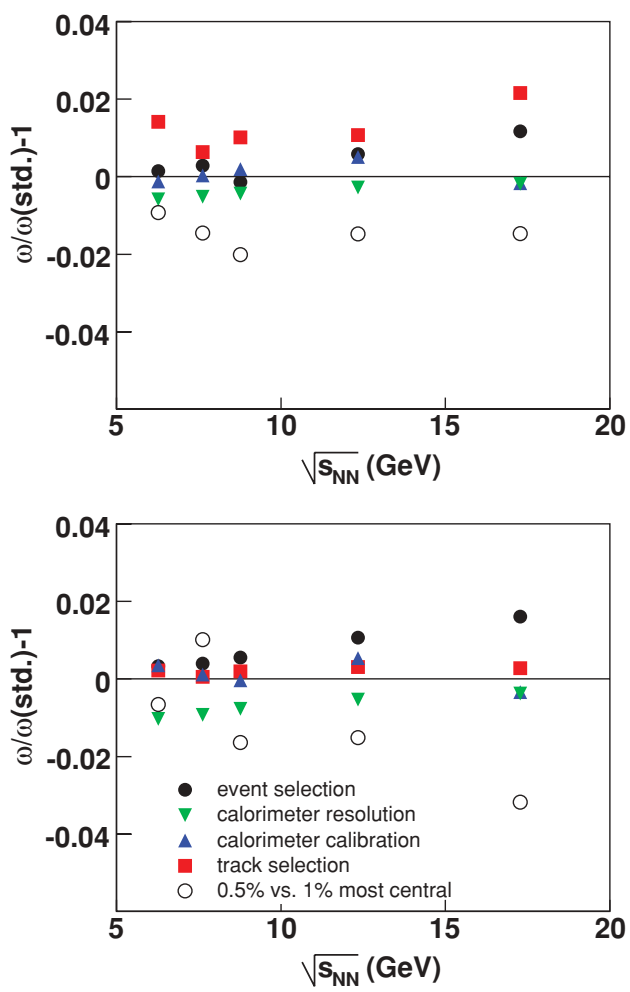

FIG. 9. (Color online) Systematic errors and difference between the $0.5 \%$ and the $1 \%$ most central collisions of the scaled variance $\omega$ of the multiplicity distribution for positively charged hadrons at midrapidity $[0<y(\pi)<1$, top] and forward acceptance $[1<$ $y(\pi)<y_{\text {beam }}$, bottom] as a function of collision energy. $\omega$ (std.) corresponds to the value obtained when using the standard event and track selection criteria and no correction for the veto calorimeter resolution. 

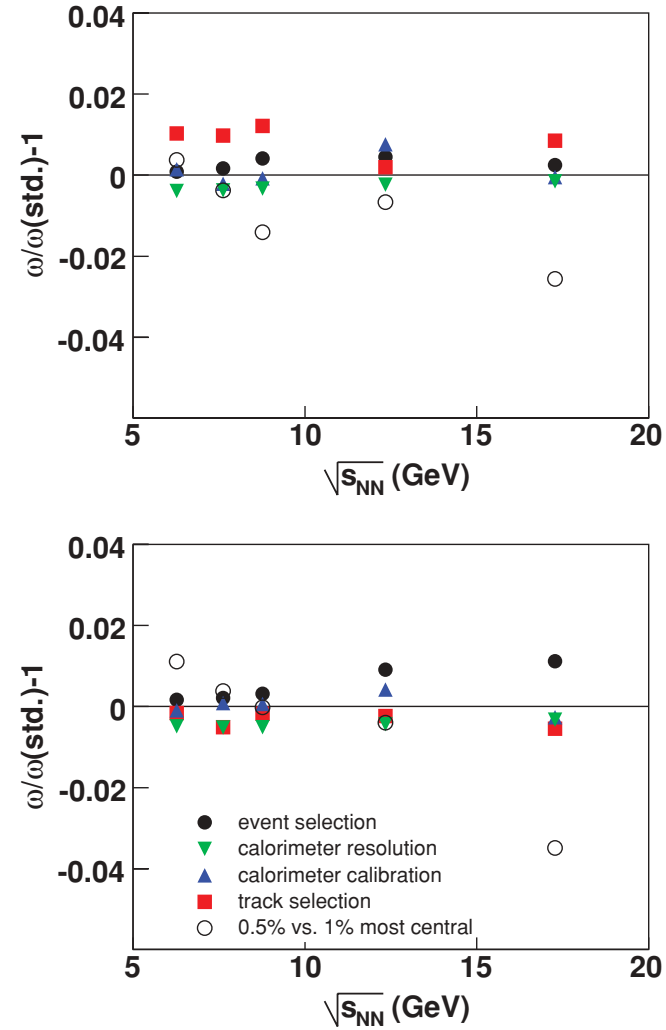

FIG. 10. (Color online) Same as Fig. 9, but for negatively charged hadrons.
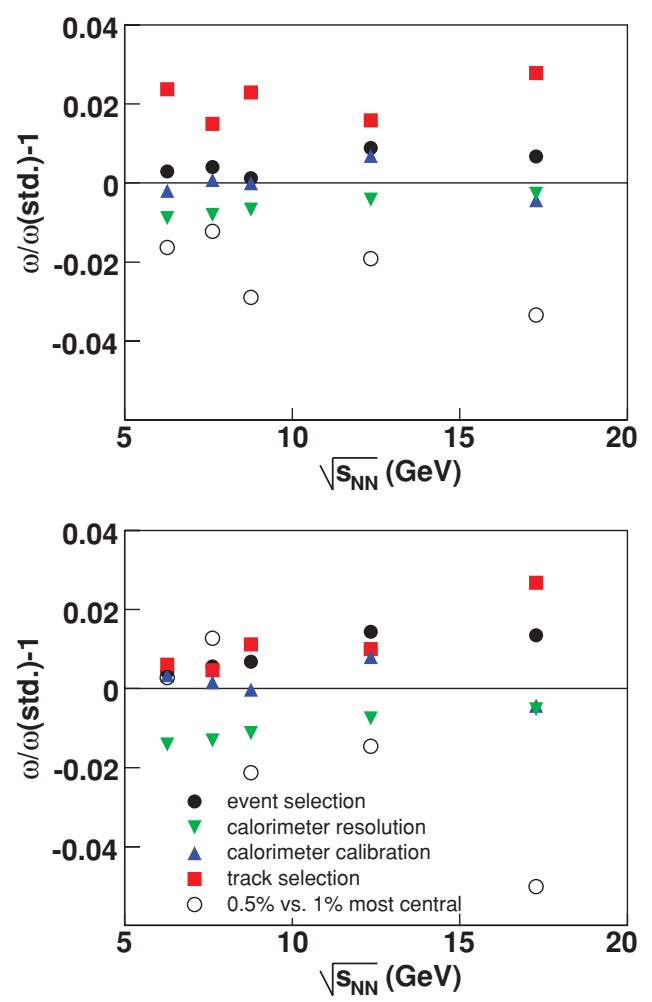

FIG. 11. (Color online) Same as Fig. 9, but for all charged hadrons.

\section{RESULTS ON MULTIPLICITY FLUCTUATIONS}

In this section, results on multiplicity fluctuations for negatively, positively, and all charged hadrons are presented for $\mathrm{Pb}+\mathrm{Pb}$ collisions at $20 A, 40 A, 80 A$, and $158 A \mathrm{GeV}$. To minimize the fluctuations in the number of participants, the $1 \%$ most central collisions according to the energy of projectile spectators measured in the veto calorimeter are selected (see Sec. III B). The rapidity interval $0<y(\pi)<y_{\text {beam }}$ used for this analysis is divided into two subintervals: $0<y(\pi)<1$ (midrapidity) and $1<y(\pi)<y_{\text {beam }}$ (forward rapidity, see Sec. III C).

In the following figures, the errors indicated by vertical lines with attached horizontal bars correspond to the statistical errors only; the thick horizontal bars are the statistical and systematic errors added in quadrature.

\section{A. Multiplicity distributions}

The multiplicity distributions for the different energies, charges, and rapidity intervals as well as the ratios of the measured multiplicity distributions to a Poisson distribution with the same mean multiplicity are shown in Figs. 12-20. For the ratio to the Poisson distributions, only points with statistical errors smaller than $20 \%$ are shown. All multiplicity distributions have a bell-like shape, and no significant tails or events with a very high or very low multiplicity are observed. The ratios of measured multiplicity distributions to the corresponding Poisson distributions are symmetric around their mean value.

The measured multiplicity distributions are narrower than the Poisson ones in the forward acceptance for positively and negatively charged hadrons at all energies. In the midrapidity acceptance, the measured distributions are wider or similar to the Poisson ones. The distributions for all charged hadrons are broader than the ones for positively and negatively charged particles separately.

\section{B. Energy dependence of $\omega$}

The energy dependence of the scaled variance $\omega$ of the multiplicity distributions for negatively, positively and all charged particles for three rapidity intervals is shown in Figs. 21-23, the numerical values are given in Table V. For positively and negatively charged hadrons, the values of $\omega$ are similar and smaller than 1 in the very forward region $\left[1<y(\pi)<y_{\text {beam }}\right]$ at all energies. At midrapidity, they are larger than 1. For all charged particles, $\omega$ is larger than for each charge separately.

No significant structure or nonmonotonic behavior is observed in the energy dependence of $\omega$.

Signatures of the critical point are expected to occur mostly at low transverse momenta [13]. The energy dependence of multiplicity fluctuations for low transverse momentum particles is shown in Fig. 24. No nonmonotonic behavior is observed. 
TABLE V. Scaled variance of the multiplicity distribution of positively, negatively, and all charged hadrons as a function of energy. The first error is statistical; the second error is systematic.

\begin{tabular}{|c|c|c|c|}
\hline \multirow[t]{2}{*}{ Energy $(\mathrm{GeV})$} & \multicolumn{3}{|c|}{$\omega\left(h^{+}\right)$} \\
\hline & $0<y(\pi)<y_{\text {beam }}$ & $0<y(\pi)<1$ & $1<y(\pi)<y_{\text {beam }}$ \\
\hline $20 A$ & $0.88 \pm 0.02 \pm 0.02$ & $0.99 \pm 0.02 \pm 0.02$ & $0.86 \pm 0.02 \pm 0.02$ \\
\hline $30 A$ & $0.85 \pm 0.01 \pm 0.02$ & $0.96 \pm 0.02 \pm 0.02$ & $0.84 \pm 0.01 \pm 0.02$ \\
\hline $40 A$ & $0.89 \pm 0.01 \pm 0.02$ & $1.01 \pm 0.01 \pm 0.02$ & $0.87 \pm 0.01 \pm 0.02$ \\
\hline $80 A$ & $0.93 \pm 0.03 \pm 0.02$ & $1.04 \pm 0.03 \pm 0.03$ & $0.89 \pm 0.03 \pm 0.02$ \\
\hline \multirow[t]{2}{*}{$158 A$} & $0.89 \pm 0.02 \pm 0.02$ & $\begin{array}{l}1.00 \pm 0.02 \pm 0.02 \\
\omega\left(h^{-}\right)\end{array}$ & $0.84 \pm 0.02 \pm 0.02$ \\
\hline & $0<y(\pi)<y_{\text {beam }}$ & $0<y(\pi)<1$ & $1<y(\pi)<y_{\text {beam }}$ \\
\hline $20 A$ & $0.94 \pm 0.02 \pm 0.02$ & $1.01 \pm 0.02 \pm 0.02$ & $0.93 \pm 0.02 \pm 0.02$ \\
\hline $30 A$ & $0.91 \pm 0.01 \pm 0.02$ & $1.01 \pm 0.02 \pm 0.02$ & $0.91 \pm 0.01 \pm 0.02$ \\
\hline $40 A$ & $0.92 \pm 0.01 \pm 0.02$ & $1.02 \pm 0.01 \pm 0.02$ & $0.91 \pm 0.01 \pm 0.02$ \\
\hline $80 A$ & $0.88 \pm 0.03 \pm 0.02$ & $1.05 \pm 0.03 \pm 0.02$ & $0.86 \pm 0.03 \pm 0.02$ \\
\hline \multirow[t]{2}{*}{$158 A$} & $0.90 \pm 0.02 \pm 0.02$ & $\begin{array}{l}1.05 \pm 0.02 \pm 0.02 \\
\quad \omega\left(h^{ \pm}\right)\end{array}$ & $0.83 \pm 0.02 \pm 0.01$ \\
\hline & $0<y(\pi)<y_{\text {beam }}$ & $0<y(\pi)<1$ & $1<y(\pi)<y_{\text {beam }}$ \\
\hline $20 A$ & $1.01 \pm 0.02 \pm 0.04$ & $1.10 \pm 0.02 \pm 0.04$ & $0.94 \pm 0.02 \pm 0.04$ \\
\hline $30 A$ & $1.01 \pm 0.02 \pm 0.04$ & $1.07 \pm 0.02 \pm 0.04$ & $0.94 \pm 0.01 \pm 0.04$ \\
\hline $40 A$ & $1.10 \pm 0.01 \pm 0.04$ & $1.15 \pm 0.01 \pm 0.04$ & $1.01 \pm 0.01 \pm 0.04$ \\
\hline $80 A$ & $1.21 \pm 0.04 \pm 0.05$ & $1.22 \pm 0.04 \pm 0.05$ & $1.07 \pm 0.03 \pm 0.04$ \\
\hline $158 A$ & $1.24 \pm 0.03 \pm 0.05$ & $1.20 \pm 0.02 \pm 0.05$ & $1.09 \pm 0.02 \pm 0.04$ \\
\hline
\end{tabular}

\section{Rapidity dependence of $\omega$}

The rapidity dependence of the scaled variance $\omega$ of the multiplicity distributions for $20 A, 30 A, 40 A, 80 A$, and $158 A \mathrm{GeV}$ central $\mathrm{Pb}+\mathrm{Pb}$ collisions is shown in Figs. 25-27. To remove the "trivial" dependence of $\omega$ on the fraction of accepted tracks [see Eq. (3)] the rapidity bins $y_{c}-\Delta y<$ $y<y_{c}+\Delta y$ are constructed in such a way that the mean multiplicity in each bin is the same.

If there were no correlations in momentum space and the single particle spectra are independent of particle multiplicity, the resulting values of $\omega$ shown in Figs. 25-27 would be independent of rapidity. This is not the case, the experimental data show an increase of $\omega$ toward midrapidity for all charges and energies.

\section{Transverse momentum dependence of $\omega$}

The transverse momentum dependence of $\omega$ at top SPS energy is shown in Fig. 28. The transverse momentum range of $0-1.5 \mathrm{GeV} / c$ is divided into five bins in such a way that the mean multiplicity in each bin is the same. The horizontal position of the points in Fig. 28 correspond to the center of gravity of the transverse momentum distribution in the transverse momentum range of the corresponding bin. Only a small rapidity interval in the forward acceptance $[1.25<y(\pi)<1.75]$ is used for this study. A larger rapidity interval might cause a bias, because the acceptance in rapidity is different for different transverse momenta.
An increase of $\omega$ is found with decreasing transverse momentum, which is more pronounced for $\omega\left(h^{-}\right)$than for $\omega\left(h^{+}\right)$. Only the top SPS energy is shown, because at lower energies the azimuthal acceptance of the NA49 detector is much smaller and therefore $\omega$ would approach unity as a result of the small multiplicity.

\section{MODEL COMPARISON}

\section{A. Hadron-resonance gas model}

In a hadron-resonance gas model, an equilibrium state of hadrons and hadronic resonances is assumed. Three different statistical ensembles are considered, namely, the grand-canonical, canonical, and microcanonical ensemble, which differ by the conservation laws taken into account. In the grand-canonical ensemble, conservation laws are not obeyed on an event-by-event basis; whereas in the canonical ensemble, the total baryon number, strangeness, and electrical charge have to be conserved in each event. In the microcanonical ensemble, the total energy and momentum are conserved in addition.

In Ref. [16] the fluctuations of particle multiplicity in full phase space are calculated for these three different statistical ensembles in the infinite volume limit. The energy dependence of multiplicity fluctuations is introduced via the chemical freeze-out parameters $T$ (temperature) and $\mu_{B}$ (baryo-chemical potential), which have been determined by hadron-resonance gas model fits at all energies to the mean particle multiplicities. Quantum statistics and resonance decays are included in the model calculations. The scaled variance $\omega$ 

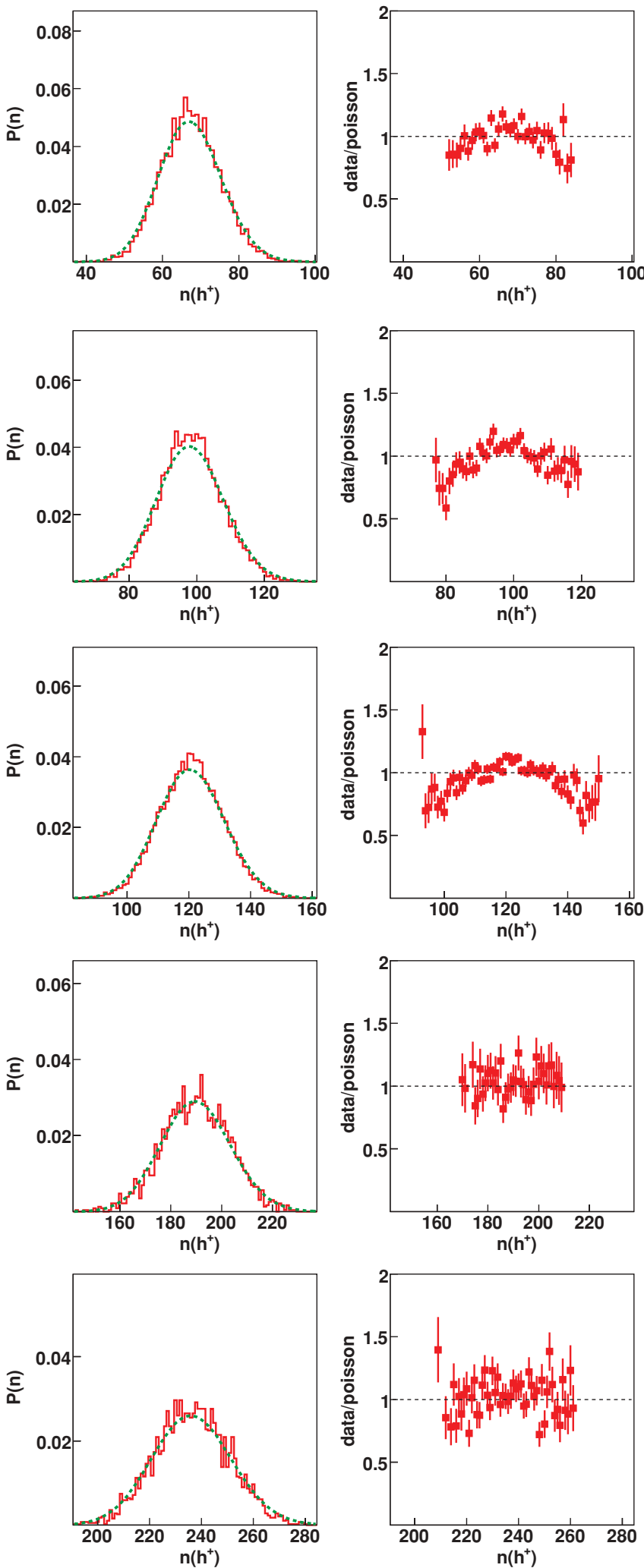

FIG. 12. (Color online) Left column: multiplicity distributions of positively charged hadrons in full experimental acceptance in the $1 \%$ most central $\mathrm{Pb}+\mathrm{Pb}$ collisions from $20 A$ (top) to $158 A \mathrm{GeV}$ (bottom). The dashed lines indicate Poisson distributions with the same mean multiplicity as in the data. Right column: the ratio of the measured multiplicity distribution to the corresponding Poisson one.
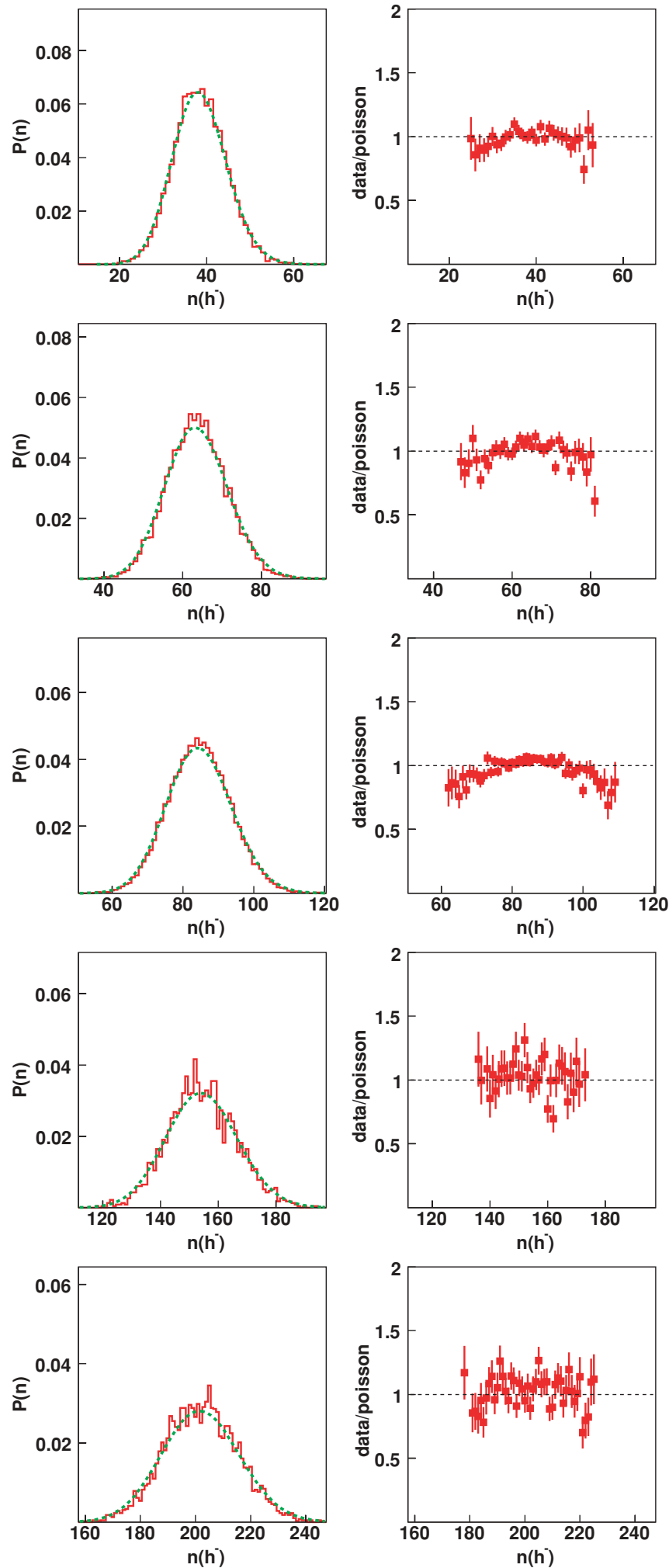

FIG. 13. (Color online) Same as in Fig. 12, but for negatively charged hadrons.

of the multiplicity distribution of negatively charged hadrons is shown in Fig. 29 as a function of collision energy.

The results for $\omega$ in the microcanonical, canonical, and grand canonical ensemble are very different at high collision 

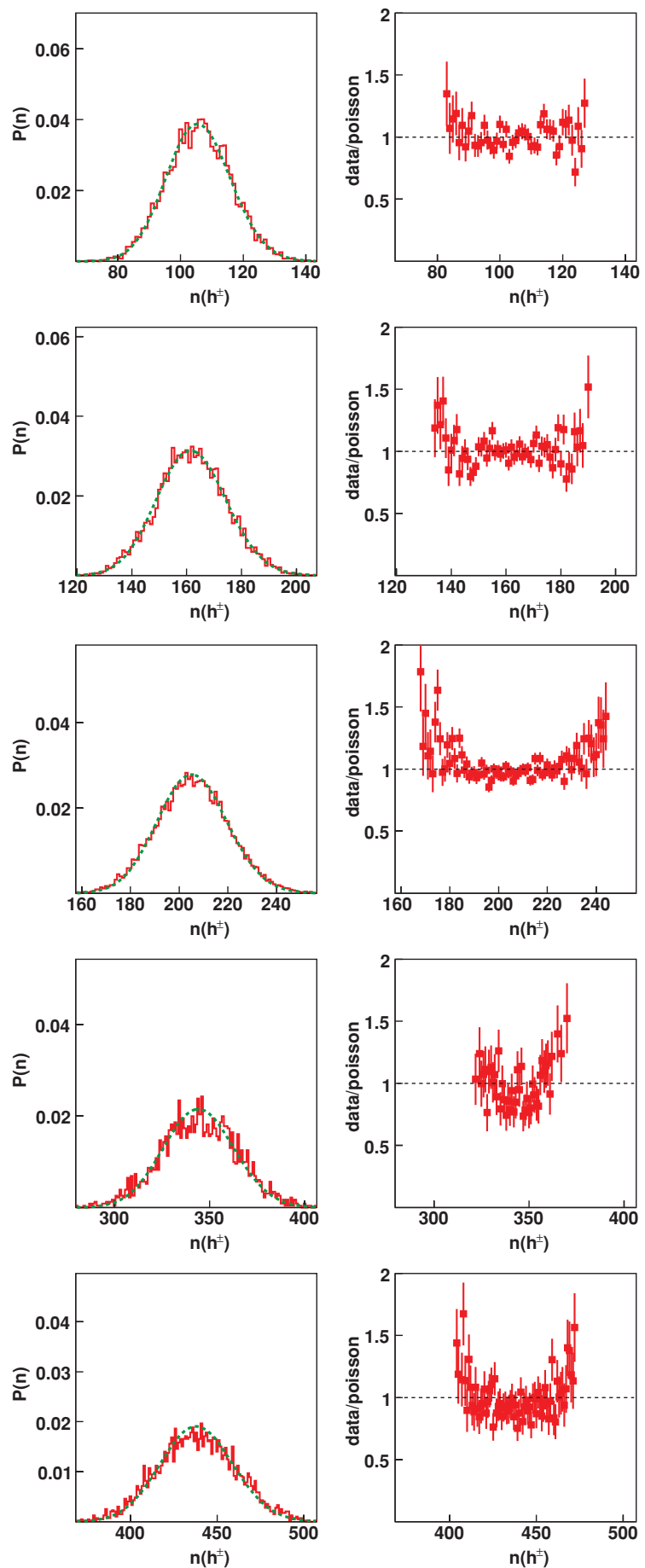

FIG. 14. (Color online) Same as in Fig. 12, but for all charged hadrons.

energies. The well-known equivalence of statistical ensembles in the large-volume limit only holds for mean values, not for multiplicity fluctuations.

The value of $\omega$ is the largest in the grand-canonical ensemble. In the microcanonical ensemble, it is the smallest; the canonical ensemble lies in between. In the canonical and microcanonical ensembles for positively and negatively charged particles separately, narrower than Poisson $(\omega<1)$
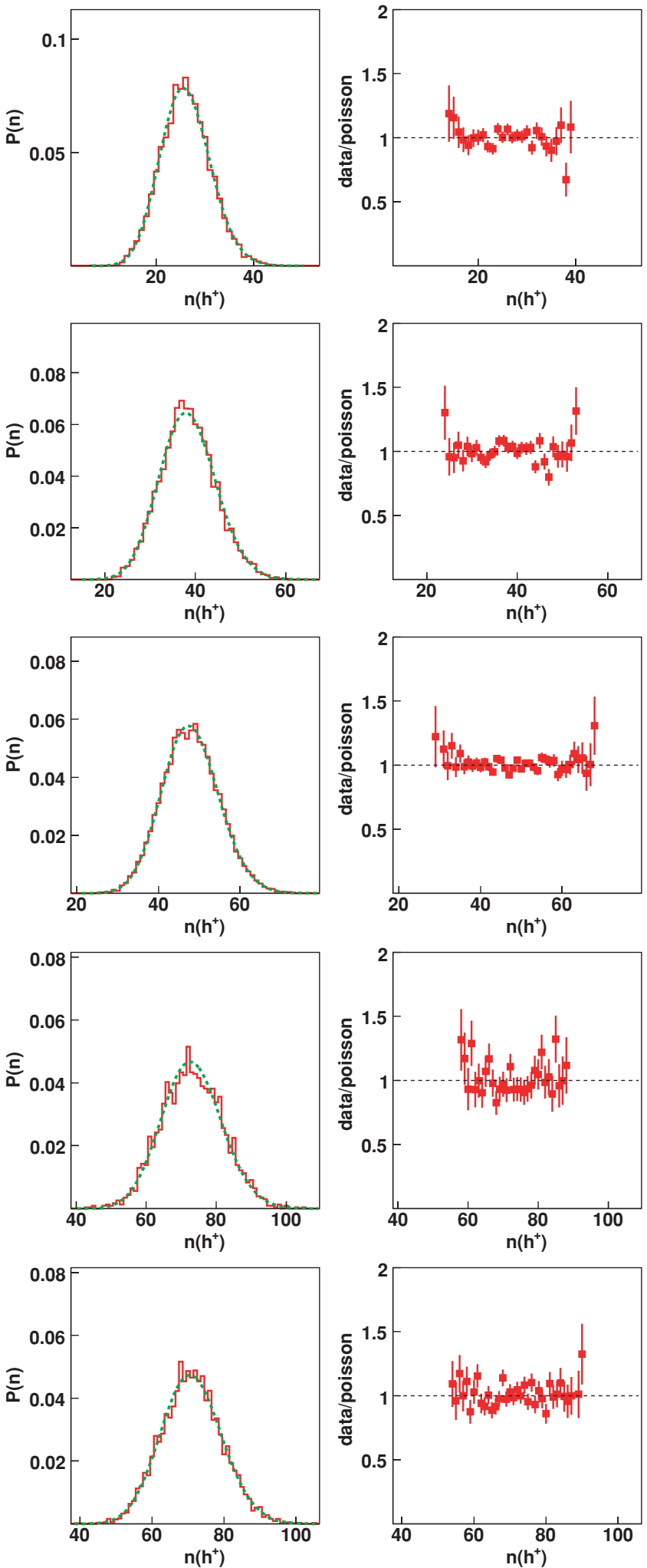

FIG. 15. (Color online) Left column: multiplicity distributions of positively charged hadrons in midrapidity acceptance in the $1 \%$ most central $\mathrm{Pb}+\mathrm{Pb}$ collisions from $20 A$ (top) to $158 \mathrm{~A} \mathrm{GeV}$ (bottom). The dashed lines indicate Poisson distributions with the same mean multiplicity as in the data. Right column: the ratio of the measured multiplicity distribution to the corresponding Poisson one. 

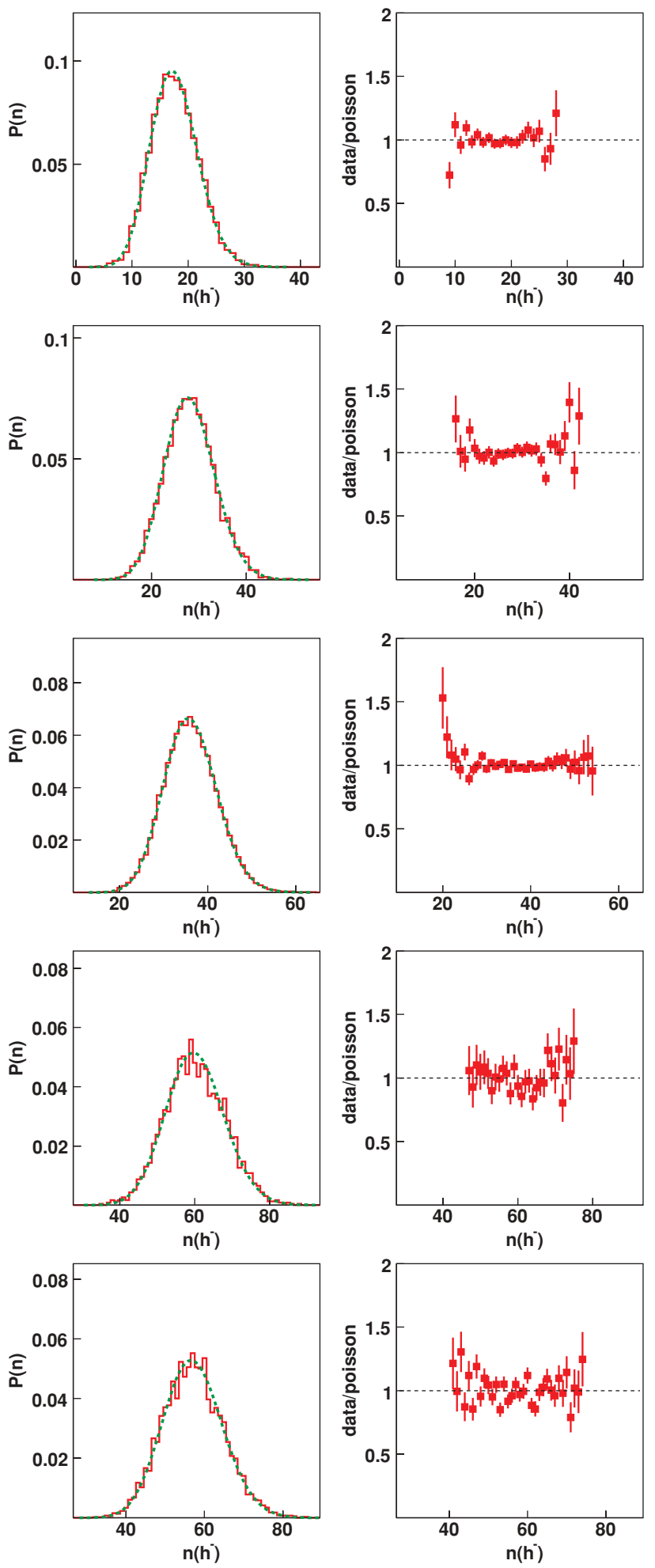

FIG. 16. (Color online) Same as Fig. 15, but for negatively charged hadrons.

multiplicity fluctuations are expected. The differences between the grand-canonical, canonical, and microcanonical ensembles show the importance of a proper treatment of conservation laws for modeling multiplicity fluctuations.

To compare the hadron-resonance gas model predictions with experimental data, we extrapolated the $\omega$ calculated
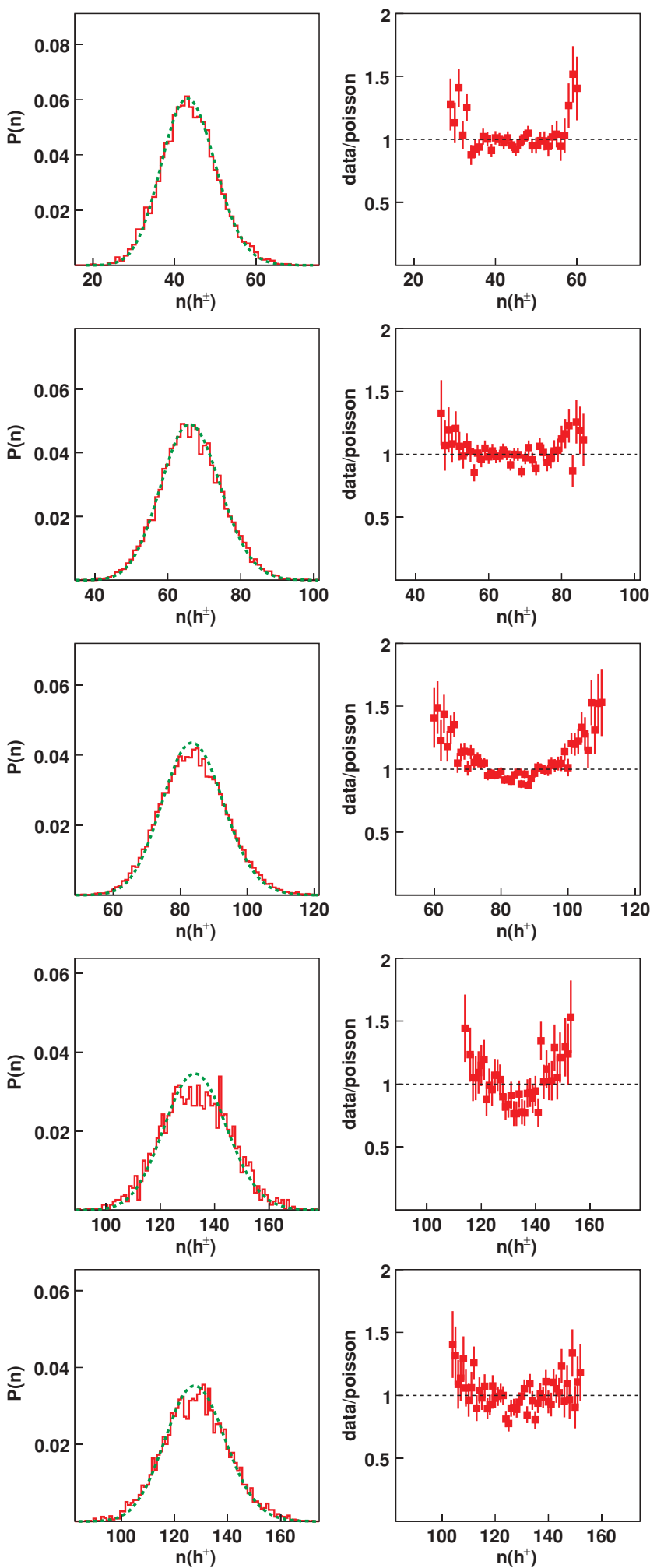

FIG. 17. (Color online) Same as Fig. 15, but for all charged hadrons.

in full phase space to the experimental acceptance using Eq. (3). Although quantum effects and resonance decays introduce correlations in momentum space, Eq. (3) is the only presently known way to compare the predictions of the grandcanonical and canonical ensembles to the experimental data. 

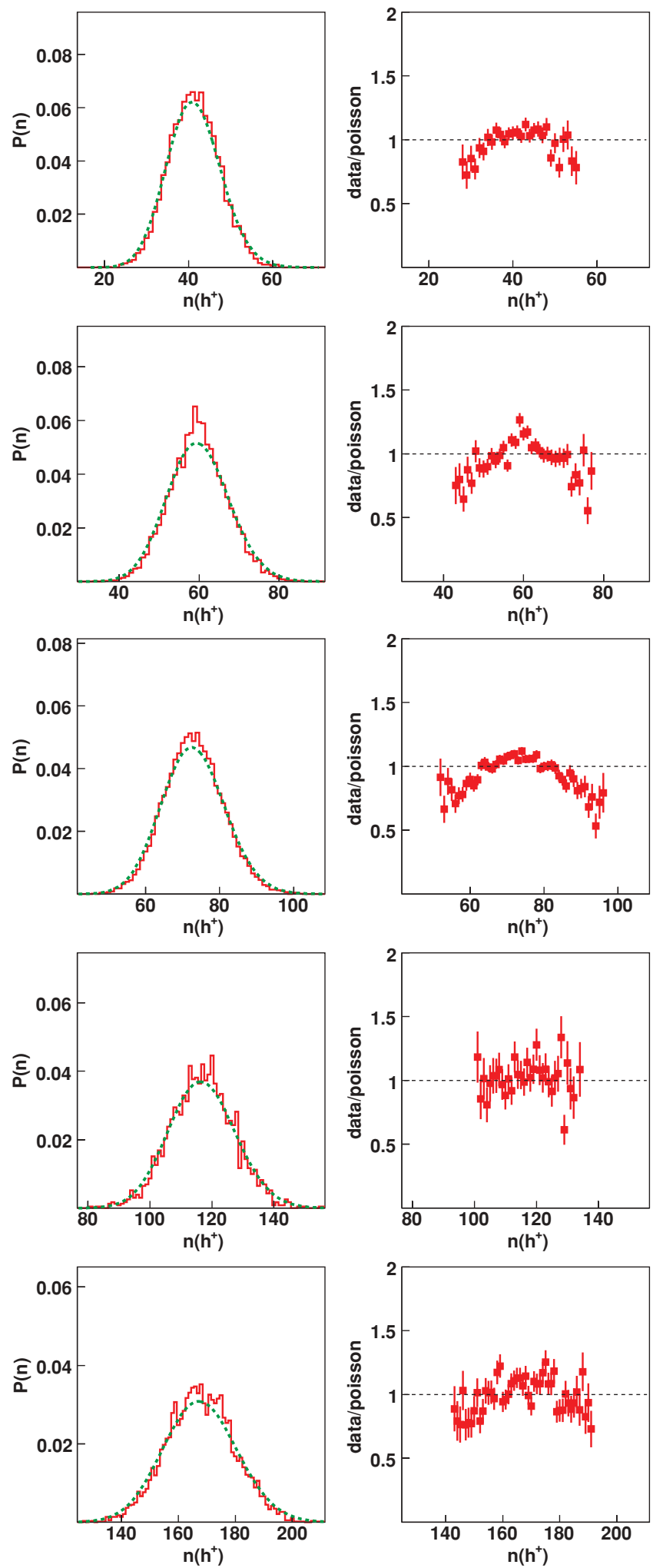

FIG. 18. (Color online) Left column: multiplicity distributions of positively charged hadrons in forward acceptance in the $1 \%$ most central $\mathrm{Pb}+\mathrm{Pb}$ collisions from $20 A$ (top) to $158 \mathrm{~A} \mathrm{GeV}$ (bottom). The dashed lines indicate Poisson distributions with the same mean multiplicity as in data. Right column: the ratio of the measured multiplicity distribution to the corresponding Poisson one.
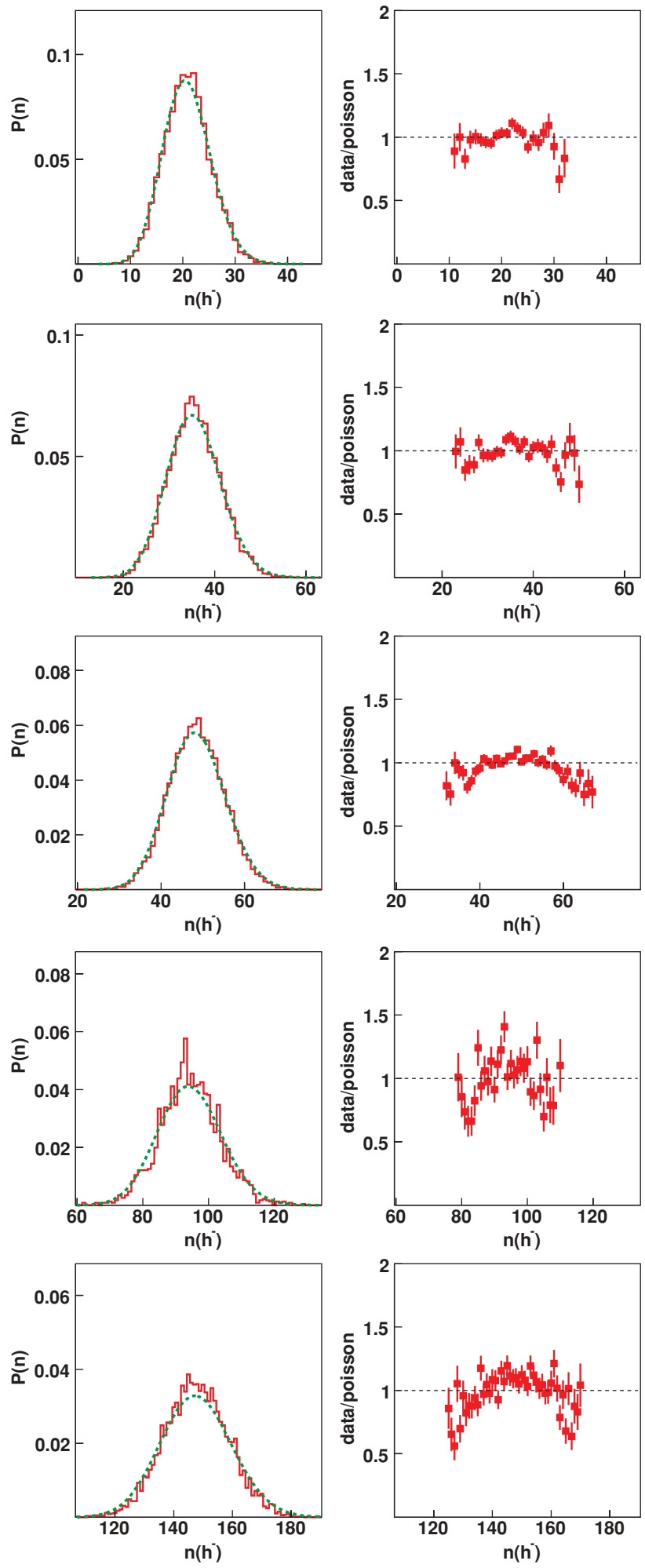

FIG. 19. (Color online) Same as Fig. 18, but for negatively charged hadrons.

For the microcanonical ensemble, the energy and momentum conservation introduces stronger correlations in momentum space [32]. Therefore Eq. (3) cannot serve as a reasonable approximation. Resonance decays introduce only a weak 

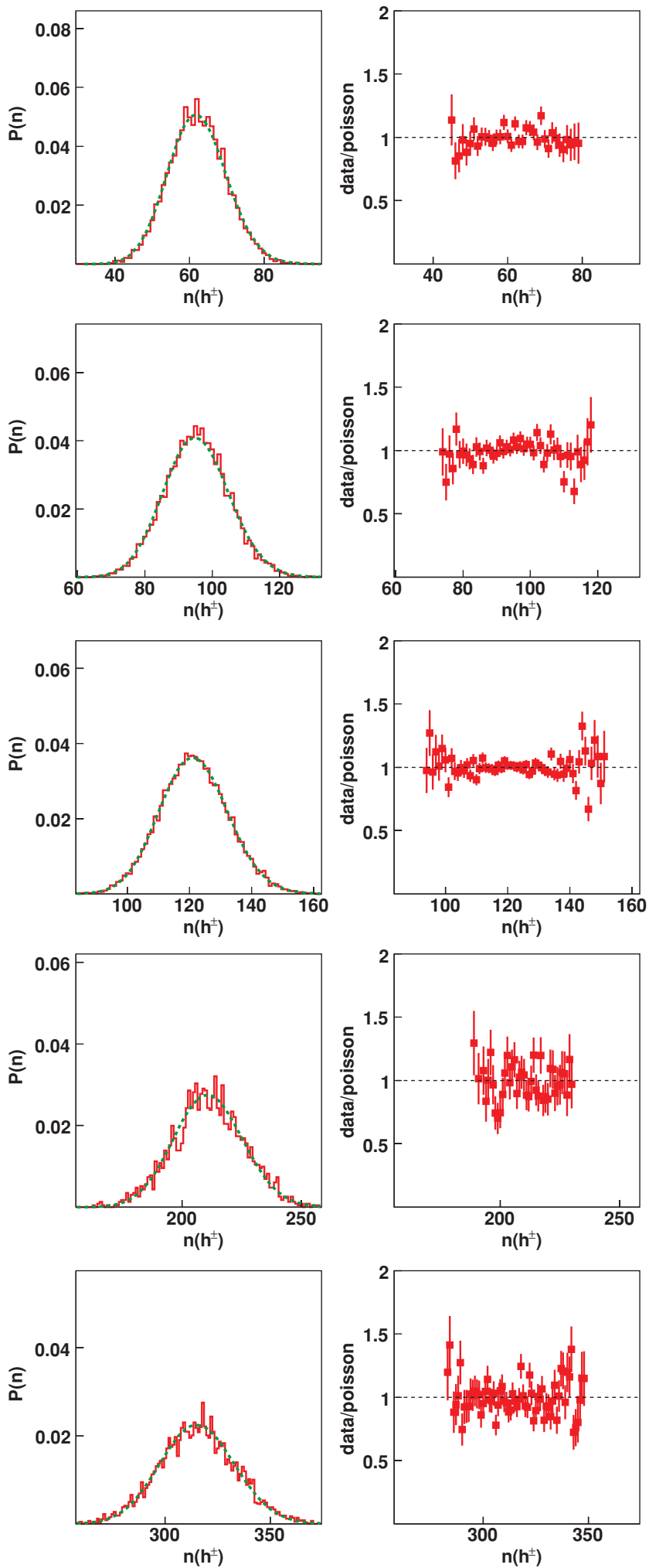

FIG. 20. (Color online) Same as Fig. 18, but for all charged hadrons.

correlation in momentum space for positively and negatively charged hadrons, because only a small number of resonances decay into two particles with the same charge. In contrast, a
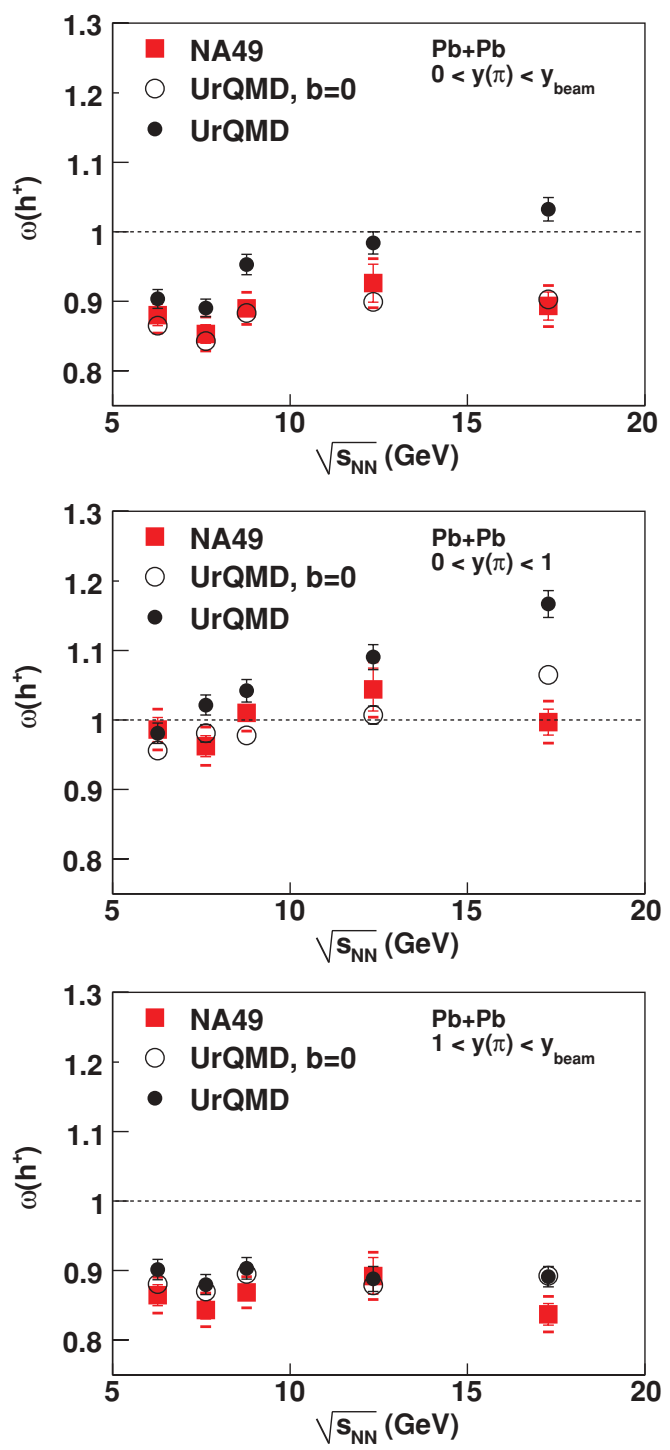

FIG. 21. (Color online) Scaled variance $\omega$ of the multiplicity distribution of positively charged hadrons produced in central $\mathrm{Pb}+\mathrm{Pb}$ collisions as a function of collision energy. Top: full experimental acceptance; middle: midrapidity; bottom: forward rapidity.

large number of resonances decay into two oppositely charged hadrons; therefore Eq. (3) is not valid for all charged hadrons.

At forward rapidity $\left[1<y(\pi)<y_{\text {beam }}\right.$ in Figs. 30 and 31], the fluctuations are overpredicted by both the canonical and the grand-canonical models. However, the canonical model is closer to the data. A microcanonical ensemble predicts smaller fluctuations than the canonical model, but a quantitative comparison with the data is not possible yet, because correlations in momentum space do not allow us to extrapolate to the experimental acceptance using Eq. (3).

At midrapidity, $\omega$ of the NA49 data (Figs. 30 and 31) is higher than in the forward region. In contrast to the experimental data, the fluctuations in the number of target participants are not included in the hadron-gas model. From comparison of UrQMD simulations for $b=0$ collisions and collisions selected according to their veto energy, it can be 

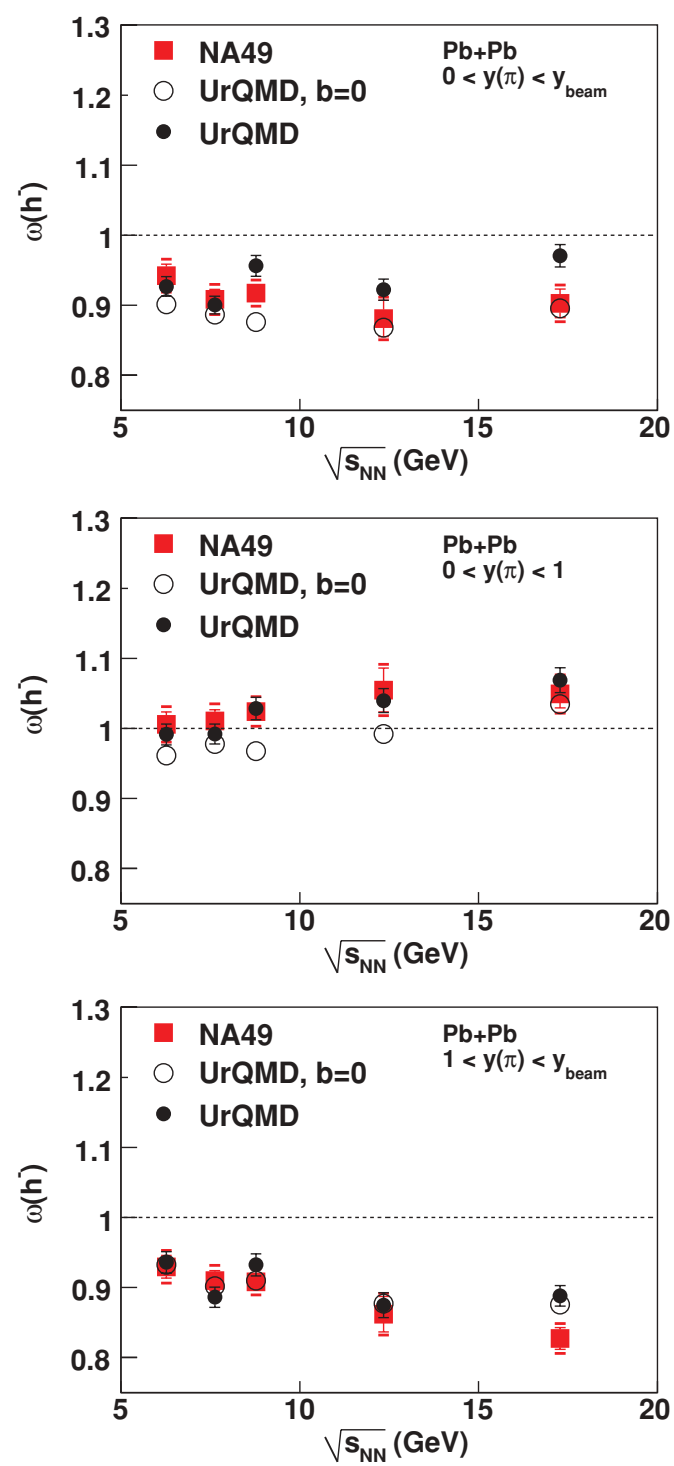

FIG. 22. (Color online) Same as Fig. 21, but for negatively charged hadrons.

estimated that the target participant fluctuations increase $\omega$ by up to $9 \%$ in the midrapidity region.

The shape of the measured multiplicity distribution is compared with the hadron-resonance gas model prediction for negatively charged hadrons at $158 \mathrm{~A} \mathrm{GeV}$ in the forward acceptance in Fig. 32. For this comparison, the multiplicity distributions for the data and the model predictions are divided by Poisson distributions with the same mean multiplicities. The hadron-resonance gas model predicts a Gaussian-like-shaped multiplicity distribution in full phase space [44]. Since this model gives no prediction about the mean multiplicity, it is taken from the data. To calculate the multiplicity distribution in the limited experimental acceptance, the distribution in the full phase space is folded with a binomial distribution accepting the same fraction $p$ of tracks as the experimental acceptance:

$$
B_{N}(n)=\frac{N !}{(N-n) ! n !} p^{n}(1-p)^{N-n}
$$
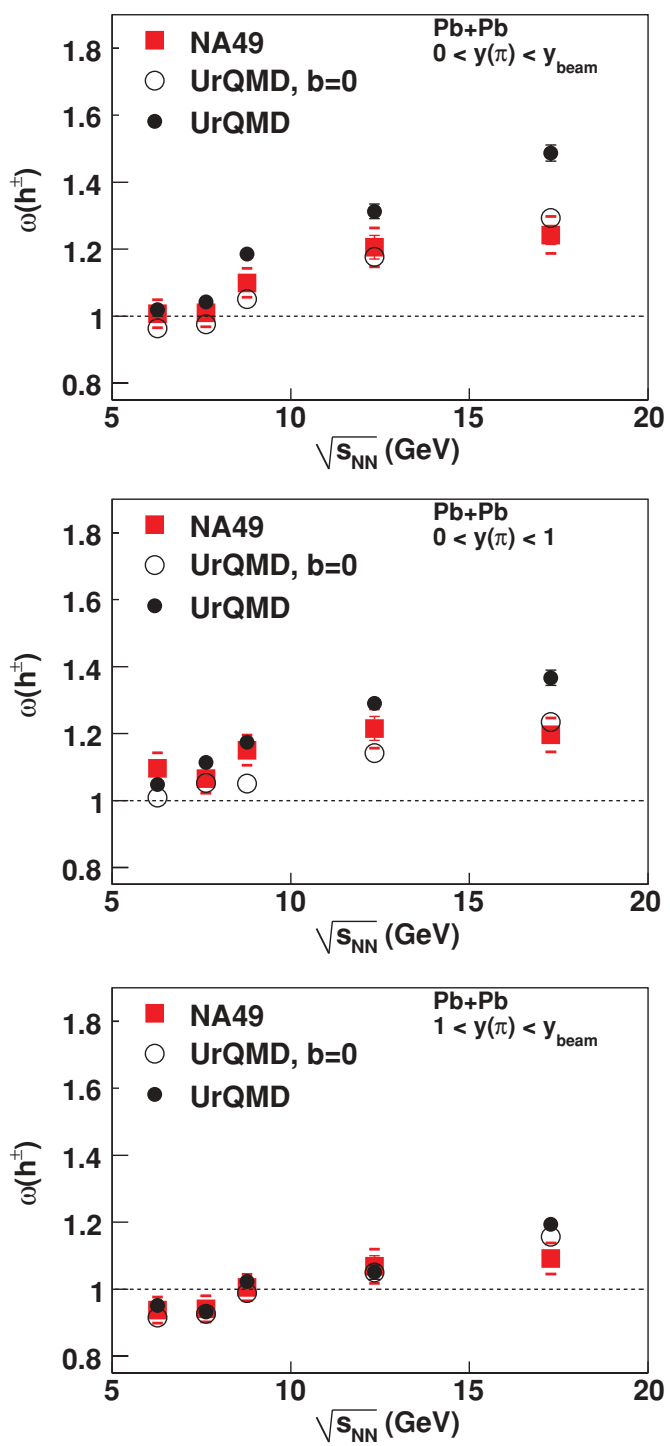

FIG. 23. (Color online) Same as Fig. 21, but for all charged hadrons.

where $N$ is the multiplicity in the full phase space and $n$ the multiplicity in the experimental acceptance. The multiplicity distribution in the experimental acceptance is given by

$$
P_{\mathrm{acc}}(n)=\sum_{N} P_{4 \pi}(N) B_{N}(n) .
$$

Note that this procedure assumes that there are no correlations in momentum space.

The ratio for the grand-canonical ensemble has a concave shape, i.e., the multiplicity distribution is wider than a Poisson distribution. For the canonical ensemble the shape is convex, showing that the distribution is narrower. The shape for the experimental data is more convex, demonstrating that the measured multiplicity distribution is even narrower than the canonical one.

In the canonical and grand-canonical ensembles of the hadron-resonance gas model, no mechanisms are present that would introduce a strong dependence of multiplicity fluctuations on rapidity or transverse momentum, which is 

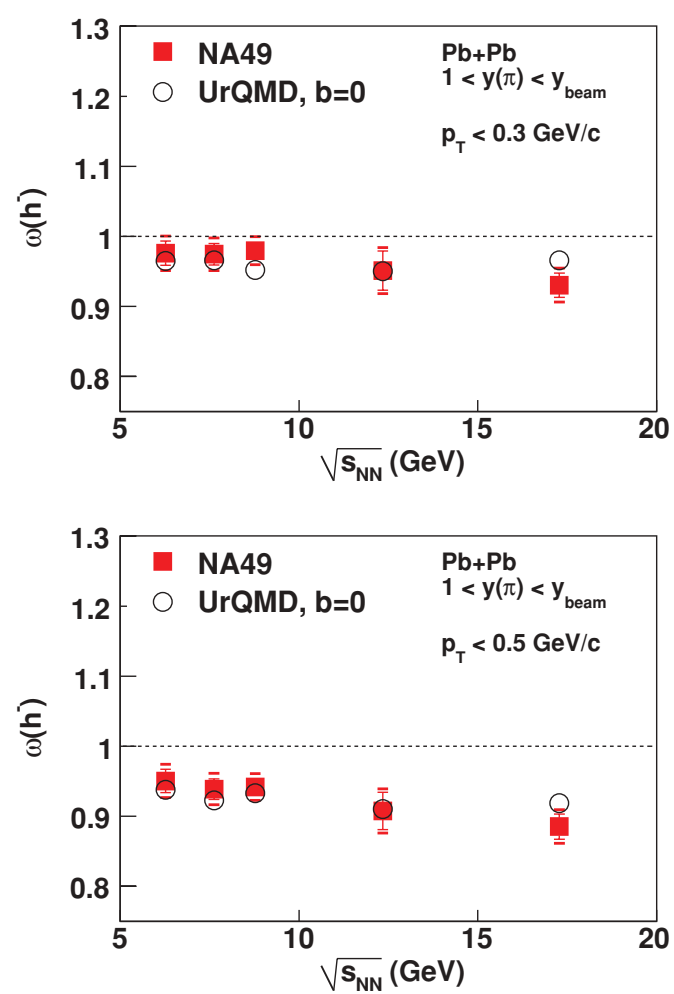

FIG. 24. (Color online) Scaled variance $\omega$ of the multiplicity distribution of negatively charged hadrons with low transverse momentum at forward rapidities produced in central $\mathrm{Pb}+\mathrm{Pb}$ collisions as a function of collision energy.

observed in the data and in UrQMD (Figs. 25-28). In a three-pion gas statistical model using the microcanonical ensemble, an increase of fluctuations near midrapidity and for low $p_{T}$ was observed [32] as an effect of energy and momentum conservation.

\section{B. String-hadronic models}

In this section, the experimental data on multiplicity fluctuations are compared with the outcome of string-hadronic model calculations, namely, of the ultrarelativistic quantum molecular dynamics model (UrQMD v1.3) [28,45] and the hadron-string dynamics model (HSD) [46].

The UrQMD microscopic transport approach is based on the propagation of constituent quarks and diquarks accompanied by mesonic and baryonic degrees of freedom. It simulates multiple interactions of in-going and newly produced particles, the excitation and fragmentation of color strings, and the formation and decay of hadronic resonances. Toward higher energies, the treatment of subhadronic degrees of freedom is of major importance. A phase transition to a quark-gluon state is not incorporated explicitly into the model dynamics.

The scaled variance $\omega$ of the multiplicity distribution of negatively charged hadrons for all inelastic $p+p$ and $p+$ $n$ interactions as well as central $(b=0) \mathrm{Pb}+\mathrm{Pb}$ collisions predicted by the UrQMD model [17] is shown in Fig. 33 as a function of the collision energy.
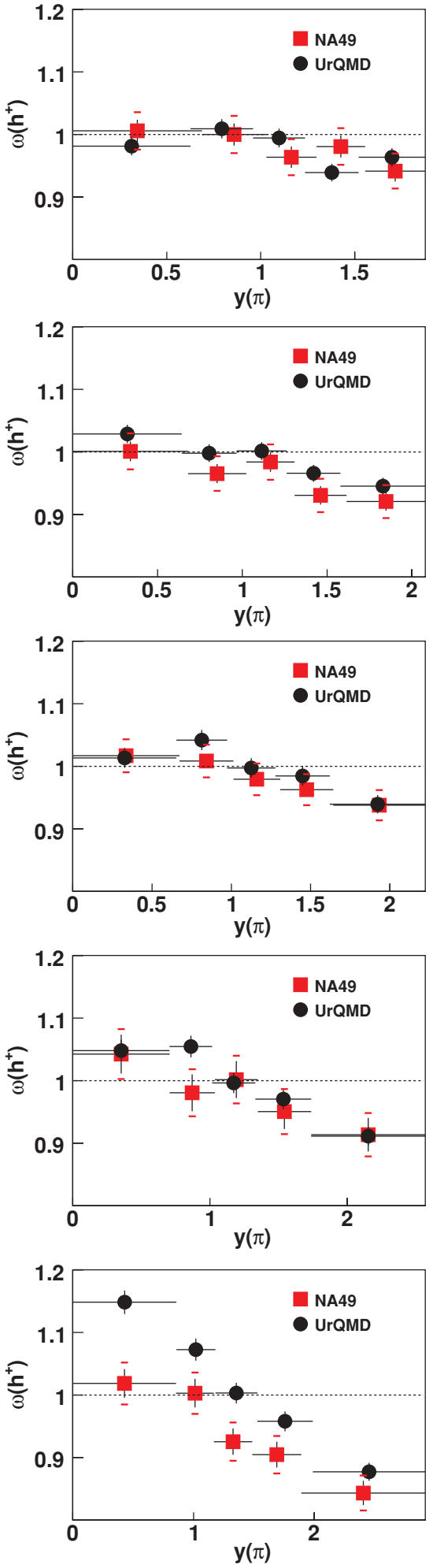

FIG. 25. (Color online) Rapidity dependence of the scaled variance $\omega$ of the multiplicity distribution of positively charged hadrons in central $\mathrm{Pb}+\mathrm{Pb}$ collisions at $20 A$ (top), $30 A, 40 A, 80 A$, and $158 A$ $\mathrm{GeV}$ (bottom) compared with UrQMD predictions with a centrality selection similar to the one for the experimental data. The rapidity bins are constructed in such a way that the mean multiplicity in each bin is the same. 

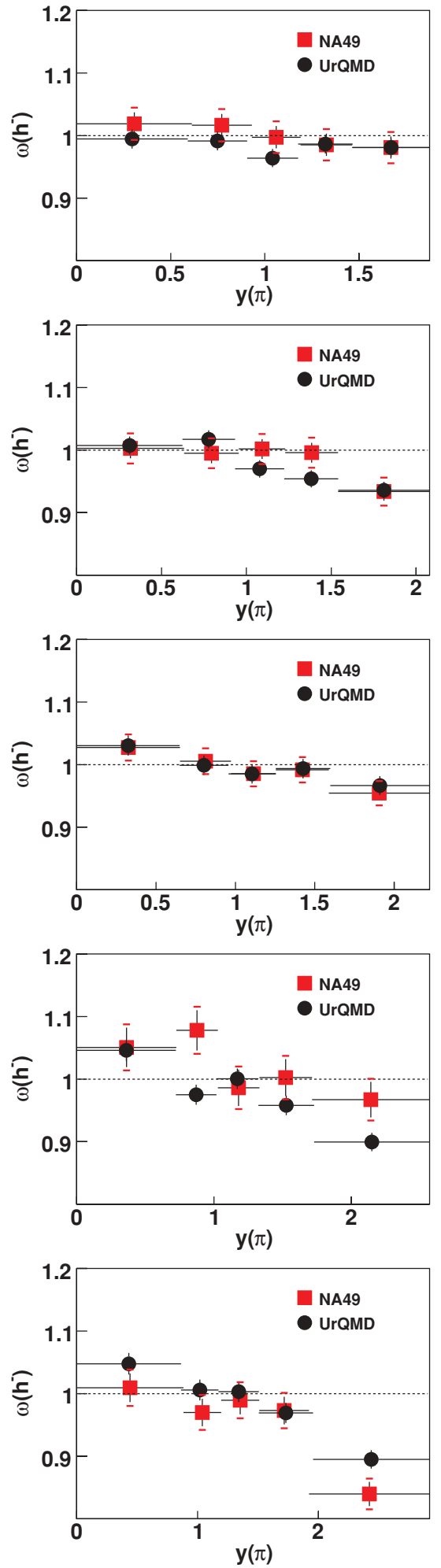

FIG. 26. (Color online) Same as Fig. 25, but for negatively charged hadrons.

The scaled variance of multiplicity fluctuations is similar in nucleon-nucleon interactions and central heavy ion collisions. Thus with respect to the scaled variance of multiplicity distributions, UrQMD behaves like a superposition model. The energy dependence of $\omega$ is different from the predictions
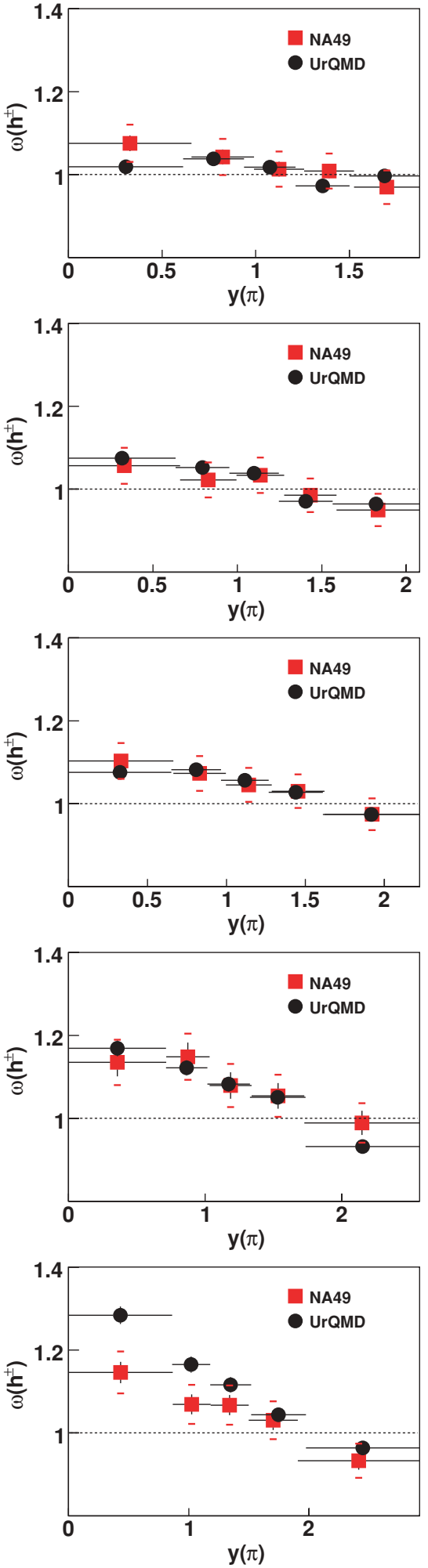

FIG. 27. (Color online) Same as Fig. 25, but for all charged hadrons.

of the hadron-resonance gas model. $\omega$ in UrQMD shows a strong increase with collision energy in accordance with the experimental $p+p$ data, while the hadron-resonance gas model has a much weaker energy dependence. 

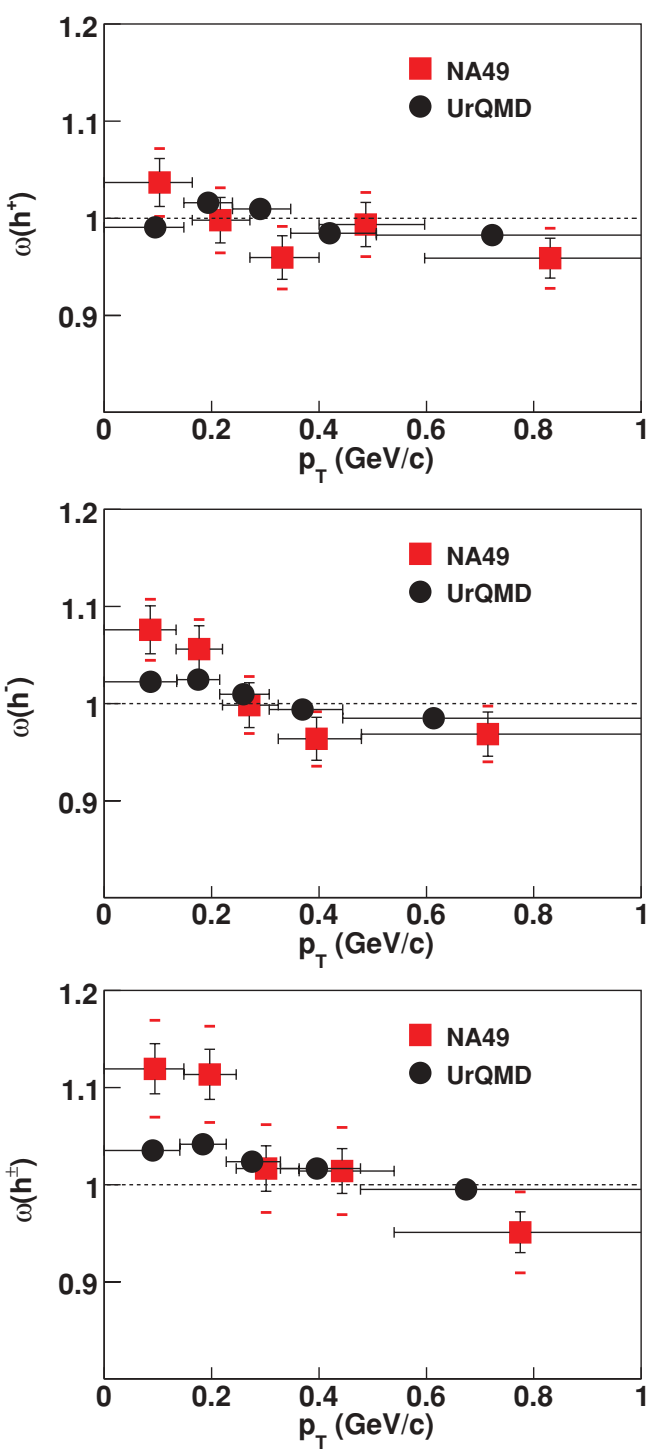

FIG. 28. (Color online) Transverse momentum dependence of the scaled variance of the multiplicity distribution of positively, negatively, and all charged hadrons in the rapidity interval $1.25<$ $y(\pi)<1.75$ in central $\mathrm{Pb}+\mathrm{Pb}$ collisions at $158 \mathrm{~A} \mathrm{GeV}$.

To compare the UrQMD model to the experimental data, both the acceptance and the centrality selection of the NA49 experiment have to be taken into account. The predictions of the model, published in Ref. [17], are compared with the experimental data in Figs. 21-23.

Two different centrality selections (see Sec. III B) are used in the model: first, collisions with zero impact parameter (open circles); second, the $1 \%$ most central collisions selected in the same way as done in the experimental data using a simulation of the acceptance of the veto calorimeter (full dots).

The UrQMD model with collisions selected by their energy in the veto calorimeter is mostly in agreement with data for all energies, acceptances, and charges. UrQMD simulation of events with zero impact parameter $(b=0)$ gives similar results in the forward rapidity region, whereas $\omega$ is smaller in the midrapidity and the full experimental regions, probably because of target participant fluctuations, which are still

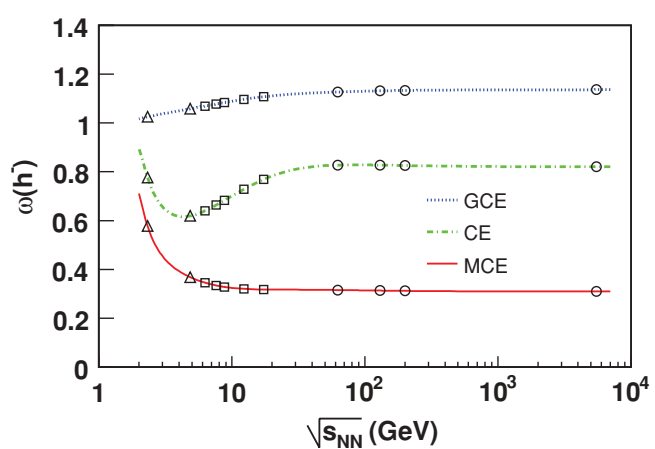

FIG. 29. (Color online) Predictions of a hadron-resonance gas model for the scaled variance $\omega$ of the multiplicity distribution in full phase space for negatively charged hadrons. The parameters of the ensemble $\left(T, \mu_{B}\right)$ are the values of the chemical freeze-out obtained by a hadron-gas model fit to produced particle ratios at different energies. Results are shown for the grand-canonical (GCE), canonical (CE), and microcanonical ensemble (MCE). The plot is taken from Ref. [16].

present for events selected by their forward-going energy, but not for collisions with a zero impact parameter.

The deviation of the multiplicity distribution from a Poisson distribution is similar in the model and in the data (see Fig. 32), but the mean multiplicity is overpredicted in the UrQMD model for all rapidity intervals, charges, and
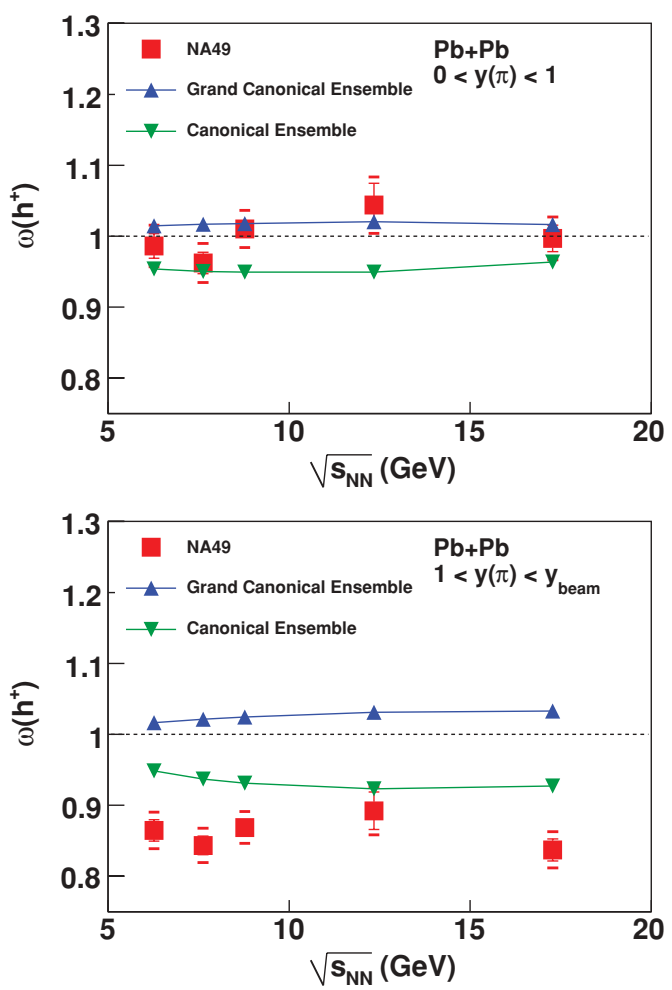

FIG. 30. (Color online) Scaled variance $\omega$ of the multiplicity distribution of positively charged hadrons produced in central $\mathrm{Pb}+\mathrm{Pb}$ collisions as a function of collision energy in midrapidity (top) and forward (bottom) acceptance compared with predictions of a grand-canonical and canonical ensemble [16]. 

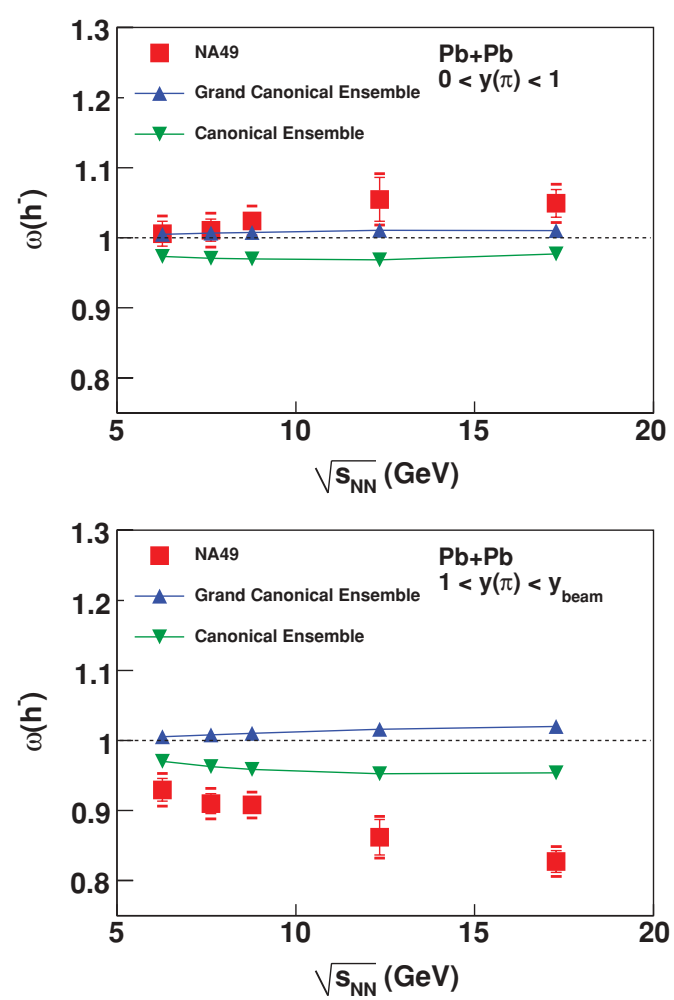

FIG. 31. (Color online) Same as Fig. 30, but for negatively charged hadrons.

energies by about $20 \%$. However, the scaled variance of the multiplicity distribution is independent of mean multiplicity for superposition models. Since it was shown that UrQMD behaves like a superposition model for $\omega$, it is justified to compare $\omega$ for data and UrQMD even though the mean multiplicities are different. Within this framework, one might

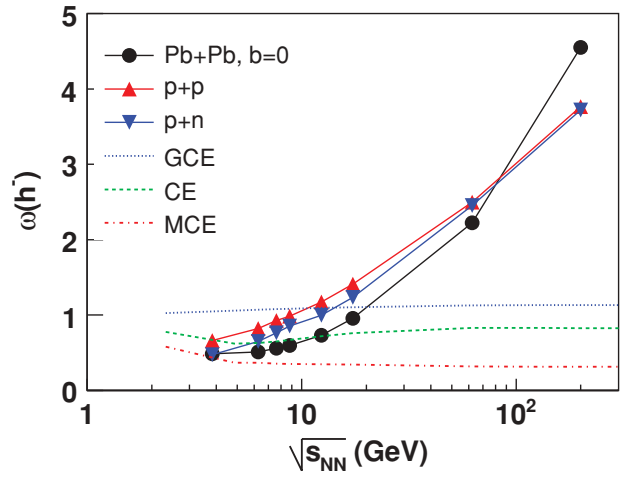

FIG. 33. (Color online) UrQMD results of scaled variance $\omega$ of negatively charged hadrons in full phase space in inelastic $p+p$, $p+n$ interactions and central $\mathrm{Pb}+\mathrm{Pb}$ collisions as a function of collision energy compared with hadron-resonance gas model predictions [16] for $\mathrm{Pb}+\mathrm{Pb}$ collisions. The plot is taken from Ref. [17].

speculate that the particle production sources in UrQMD are properly modeled, but the number of sources is overestimated in central $\mathrm{Pb}+\mathrm{Pb}$ collisions.

In the experimental data, an increase of fluctuations is observed when approaching midrapidity (Figs. 25-27). The UrQMD model reproduces this behavior when a similar centrality selection is used as in the data.

For the data, an increase of $\omega$ is measured with decreasing transverse momentum at forward rapidity (Fig. 28). In the UrQMD model, a similar trend is observed, but $\omega$ is underpredicted at low transverse momenta. This might be related to such effects as Coulomb and Bose-Einstein correlations, which are not implemented in the model.
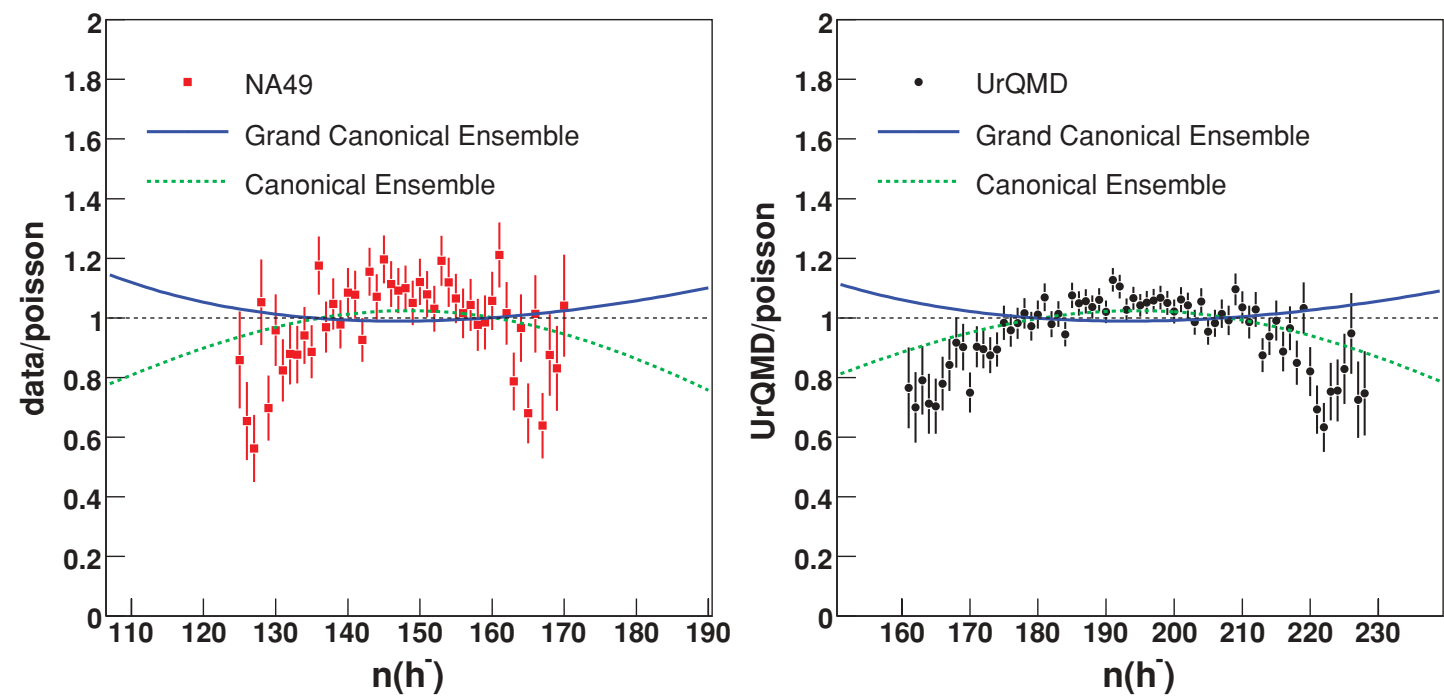

FIG. 32. (Color online) Ratio of multiplicity distribution of NA49 experimental data and UrQMD simulation to a Poisson distribution with the same mean value for negatively charged hadrons in $\mathrm{Pb}+\mathrm{Pb}$ collisions at $158 \mathrm{~A} \mathrm{GeV}$ in the forward acceptance. Only points with statistical errors smaller than $20 \%$ are shown. Hadron-gas model predictions in the grand-canonical and canonical ensembles with the same mean multiplicity and fraction of accepted tracks are also shown. 
The HSD transport approach, following a similar strategy as the UrQMD model, yields similar results for $\omega$. The energy dependence for central $(b=0) \mathrm{Pb}+\mathrm{Pb}$ collisions obtained by the HSD model are presented in Ref. [30]. These predictions were compared with preliminary NA49 results on multiplicity fluctuations in Ref. [42] and were found to agree in the forward acceptance. Unfortunately, HSD calculations for the larger acceptance used in this paper are not yet available.

\section{Onset of deconfinement}

In heavy ion collisions, initial fluctuations in the stopped energy $E$ are expected to cause fluctuations in the entropy $S$ [12]. The energy dependences of various hadron production properties, such as the kaon to pion ratio, the inverse slope parameter of kaons, and the pion multiplicity $[10,47]$, show anomalies at low SPS energies that may be attributed to the onset of deconfinement [11]. In Ref. [12], it is predicted that this should lead to a nonmonotonic behavior of the ratio of fluctuations of entropy to stopped energy:

$$
R_{e}=\frac{(\delta S)^{2} / S^{2}}{(\delta E)^{2} / E^{2}}
$$

At intermediate SPS energies, where a mixed phase of hadron gas and QGP is assumed, a "shark-fin" structure with a maximum near $80 \mathrm{~A} \mathrm{GeV}$ is predicted. $R_{e}$ is approximately 0.6 in both the hadron and QGP phases; in the mixed phase, it can reach values up to 0.8 .

In Ref. [16], these relative fluctuations are related to multiplicity fluctuations under the assumption of a proportionality of entropy to produced particle multiplicity, namely,

$$
\omega_{\delta E} \approx \frac{(\delta E)^{2}}{E^{2}}\langle n\rangle R_{e}
$$

The fluctuations of thermalized energy are obtained by UrQMD and HSD simulations and are found to be $\delta E / E<$ 0.03 .

Using this result, one can estimate the additional multiplicity fluctuations of negatively charged hadrons caused by the fluctuations of thermalized energy to be $\omega_{\delta E}\left(h^{-}\right) \approx 0.02$ for the pure hadron gas or QGP phase. In the mixed phase, the expectation for $\omega_{\delta E}\left(h^{-}\right)$amounts to $\approx 0.03$ at $80 A \mathrm{GeV}$. The predicted increase of $\omega$ by 0.01 due the mixed phase is smaller than the systematic error on the measurement of $\omega$. Therefore the data can neither support nor disprove the existence of a mixed phase at SPS energies.

\section{First-order phase transition}

It is suggested in Ref. [29] that droplets of hadronic matter should be formed in matter when the system crosses the firstorder phase transition line during cooldown. These droplets are expected to produce multiplicity fluctuations 10-100 times larger than the Poisson expectation in the full phase space. No predictions of the increase of $\omega$ for the limited experimental acceptance are available, but naively it can be expected to be on the order of 1-10 [according to Eq. (3)].
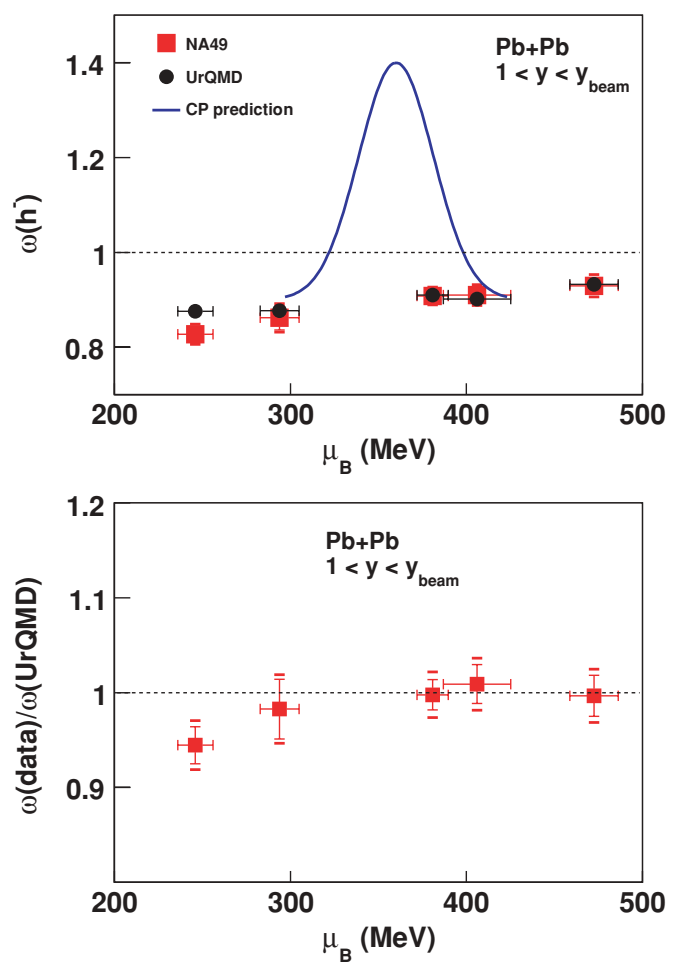

FIG. 34. (Color online) Top: Scaled variance $\omega$ of the multiplicity distribution of negatively charged hadrons at forward rapidities produced in central $\mathrm{Pb}+\mathrm{Pb}$ collisions as a function of the baryochemical potential $\mu_{B}$ [48]. A sketch of the expected increase of $\omega$ due to the critical point $[13,51]$ is also shown. The UrQMD results are given for a centrality selection similar to the experimental data. Bottom: Ratio of $\omega$ in data and UrQMD as a function of $\mu_{B}$.

In our acceptance, an excess of multiplicity fluctuations with respect to the UrQMD baseline, which does not include an explicit phase transition, of larger than 0.1 can be excluded (see Fig. 34).

\section{E. Critical point}

It is expected that the hadron gas and quark-gluon plasma regions in the phase diagram of strongly interacting matter are separated by a first-order phase transition line at high baryochemical potentials and moderate temperatures. A crossover between both phases is predicted for high temperatures and low baryo-chemical potentials. Then the first-order phase transition line will end in a critical point.

If the freeze-out of matter happens near the critical point, large fluctuations, for instance, in multiplicity and transverse momentum, are expected. In Ref. [13], it is estimated that the scaled variance of the distribution of total multiplicity of single charged hadrons should increase by about 1 near the critical point. However, this estimate has a large and difficult-to-estimate systematic error. The limited acceptance should reduce the critical point signal by a factor of about 2 . Consequently, the expected increase of the scaled variance in the vicinity of the critical point is about 0.5 . 
These critical fluctuations are expected to be located mainly at low transverse momenta [13]. The scaled variance as a function of the baryo-chemical potential is compared in Fig. 34 with the UrQMD baseline. As the increase of fluctuations due to the freeze-out in the vicinity of the critical point is expected to be restricted to a range in the baryo-chemical potential which is comparable to the difference in baryo-chemical potentials of the different collision energies [49], the signature of the critical point is expected to increase $\omega$ at one collision energy only. A sketch of the expected increase of $\omega$ due to the critical point [13] is shown in Fig. 34. No significant increase of $\omega$ that may be attributed to the critical point is observed in the data. The scaled variance for low transverse momentum particles (see Fig. 24) does not show a significant nonmonotonic structure or excess over the UrQMD baseline either.

\section{SUMMARY}

The energy dependence of multiplicity fluctuations in central $\mathrm{Pb}+\mathrm{Pb}$ collisions at $20 A, 30 A, 40 A, 80 A$, and $158 A$ $\mathrm{GeV}$ was studied for positively, negatively, and all charged hadrons. The total selected experimental acceptance $[0<$ $\left.y(\pi)<y_{\text {beam }}\right]$ is divided into a midrapidity $[0<y(\pi)<1]$ and a forward rapidity $\left[1<y(\pi)<y_{\text {beam }}\right]$ region. At forward rapidity, a suppression of fluctuations compared to a Poisson distribution is observed for positively and negatively charged hadrons. At midrapidity and for all charged hadrons, the fluctuations are higher. Furthermore, the rapidity dependence at all energies and the transverse momentum dependence at $158 \mathrm{~A} \mathrm{GeV}$ were studied. The scaled variance of the multiplicity distribution increases for decreasing rapidity and transverse momentum.

The string-hadronic UrQMD model significantly overpredicts the mean multiplicities, but it approximately reproduces the scaled variance of the multiplicity distributions.

Multiplicity fluctuations predicted by the grand-canonical and canonical formulations of the hadron-resonance gas model [16] overpredict fluctuations in the forward acceptance. The microcanonical formulation predicts smaller fluctuations and can qualitatively reproduce the increase of fluctuations for low rapidities and transverse momenta. However, no quantitative calculation is available yet for the limited experimental acceptance.

At RHIC and LHC energies, the difference in $\omega$ for the string-hadronic and the hadron-gas models in the full phase space is much larger than for SPS energies, and experimental data should be able to distinguish between them rather easily.

Narrower than Poissonian $(\omega<1)$ multiplicity fluctuations are measured in the forward kinematic region $[1<y(\pi)<$ $\left.y_{\text {beam }}\right]$. They can be related to the reduced fluctuations predicted for relativistic gases with imposed conservation laws. This general feature of relativistic gases may be preserved also for some nonequilibrium systems as modeled by the string-hadronic approaches.

The predicted maximum in fluctuations due to a firstorder phase transition from hadron-resonance gas to QGP [12] is smaller than the experimental errors of the present measurements and can therefore neither be confirmed nor disproved.

No sign of increased fluctuations as expected for a freezeout near the critical point of strongly interacting matter was observed. The future NA61 program [50] will study both the energy and system size dependence of fluctuations with improved sensitivity in a systematic search for the critical point.

\section{ACKNOWLEDGMENTS}

Fruitful discussions with M. Bleicher, M. Hauer, E. Bratkovskaya, V. Konchakovski, V. Begun, M. Gorenstein, and I. Mishustin are gratefully acknowledged. This work was supported by the U.S. Department of Energy, Grant DE-FG03-97ER41020/A000, the Bundesministerium fur Bildung und Forschung, Germany (06F137), the Virtual Institute VI-146 of Helmholtz Gemeinschaft, Germany, the Polish State Committee for Scientific Research (1 P03B 006 30, N N202 078735, 1 PO3B 121 29, 1 P03B 127 30), the Hungarian Scientific Research Foundation (T032648, T032293, T043514), the Hungarian National Science Foundation, OTKA, (F034707), the Polish-German Foundation, the Korea Science \& Engineering Foundation (R01-2005-000-10334-0), the Bulgarian National Science Fund (Ph-09/05), and the Croatian Ministry of Science, Education and Sport (Project 098-0982887-2878).

\section{APPENDIX: DERIVATIONS}

\section{Acceptance dependence of $\omega$}

Provided the particles are produced independently in momentum space and the form of the momentum distribution is independent of multiplicity, the scaled variance in a limited acceptance is related to the scaled variance in full phase space $(4 \pi)$ by an analytic formula.

Under these assumptions, having an experimental acceptance registering the fraction $p$ of the total number of tracks $N$ is equivalent to a roll of the dice for each particle in the full phase space and to accept it with a probability of $p$. Therefore the probability to measure a number of particles $n$ in a fixed acceptance follows the binomial distribution

$$
B(n \mid N)=\frac{N !}{n !(N-n) !} p^{n}(1-p)^{N-n} .
$$

For a number of particles varying in the full phase space according to $P_{4 \pi}(N)$, the probability to measure a number of particles $n$ in the limited acceptance is

$$
P_{A}(n)=\sum_{N} B(n \mid N) P_{4 \pi}(N) .
$$

From Eqs. (A1) and (A2), it follows that the mean number of particles in the acceptance is

$$
\langle n\rangle=p\langle N\rangle,
$$


and the variance of the number $n$ of particles in the acceptance is given by

$$
\begin{aligned}
\operatorname{Var}(n) & =\langle\operatorname{Var}(n \mid N)\rangle+\operatorname{Var}(\langle n \mid N\rangle) \\
& =\langle\operatorname{Var}(n \mid N)\rangle+\operatorname{Var}(p N) \\
& =\langle N\rangle p(1-p)+p^{2} \operatorname{Var}(N) .
\end{aligned}
$$

Finally, the scaled variance in the limited acceptance $\omega_{\text {acc }}$ is related to the scaled variance in the full phase space, $\omega_{4 \pi}$, as

$$
\omega_{\mathrm{acc}}=p\left(\omega_{4 \pi}-1\right)+1 .
$$

The acceptance dependence given by Eq. (A5) is not valid when effects like resonance decays, quantum statistics, and energy and momentum conservation introduce correlations in momentum space.

\section{Participant fluctuations}

In a superposition model, the multiplicity $n$ is the sum of the number of particles produced by $k$ particle-production sources,

$$
n=\sum_{i=1}^{k} n_{i}^{\text {so }}
$$

where the summation index $i$ runs over the sources. Assuming statistically identical sources the mean multiplicity is

$$
\langle n\rangle=\langle k\rangle\left\langle n^{\text {so }}\right\rangle
$$

and the variance reads

$$
\operatorname{Var}(n)=\langle k\rangle \operatorname{Var}\left(n^{\mathrm{so}}\right)+\left\langle n^{\mathrm{so}}\right\rangle^{2} \operatorname{Var}(k)
$$

Using these equations, the scaled variance of $n$ can be expressed as

$$
\omega=\frac{\langle k\rangle \operatorname{Var}\left(n^{\mathrm{so}}\right)}{\langle k\rangle\left\langle n^{\mathrm{so}}\right\rangle}+\frac{\left\langle n^{\mathrm{so}}\right\rangle^{2} \operatorname{Var}(k)}{\langle k\rangle\left\langle n^{\mathrm{so}}\right\rangle}=\omega^{\text {so }}+\left\langle n^{\mathrm{so}}\right\rangle \omega_{k} .
$$

For the case of a constant number of sources, the scaled variance is independent of the number of sources.
[1] J. C. Collins and M. J. Perry, Phys. Rev. Lett. 34, 1353 (1975).

[2] E. V. Shuryak, Phys. Rep. 61, 71 (1980).

[3] S. Margetis et al. (NA49 Collaboration), Phys. Rev. Lett. 75, 3814 (1995).

[4] U. W. Heinz and M. Jacob (2000), nucl-th/0002042.

[5] J. Adams et al. (STAR Collaboration), Nucl. Phys. A757, 102 (2005).

[6] K. Adcox et al. (PHENIX Collaboration), Nucl. Phys. A757, 184 (2005).

[7] B. B. Back et al., Nucl. Phys. A757, 28 (2005).

[8] I. Arsene et al. (BRAHMS Collaboration), Nucl. Phys. A757, 1 (2005).

[9] T. Alber et al. (NA49 Collaboration), Phys. Rev. C 66, 054902 (2002), nucl-ex/0205002.

[10] C. Alt et al. (NA49 Collaboration), Phys. Rev. C 77, 024903 (2008).

[11] M. Gazdzicki and M. I. Gorenstein, Acta Phys. Pol. B 30, 2705 (1999).

[12] M. Gazdzicki, M. I. Gorenstein, and S. Mrówczyński, Phys. Lett. B585, 115 (2004).

[13] M. A. Stephanov, K. Rajagopal, and E. V. Shuryak, Phys. Rev. D 60, 114028 (1999).

[14] V. V. Begun, M. Gazdzicki, M. I. Gorenstein, and O. S. Zozulya, Phys. Rev. C 70, 034901 (2004).

[15] V. V. Begun, M. I. Gorenstein, A. P. Kostyuk, and O. S. Zozulya, Phys. Rev. C 71, 054904 (2005).

[16] V. V. Begun, M. Gazdzicki, M. I. Gorenstein, M. Hauer, V. P. Konchakovski, and B. Lungwitz, Phys. Rev. C 76, 024902 (2007).

[17] B. Lungwitz and M. Bleicher, Phys. Rev. C 76, 044904 (2007).

[18] V. P. Konchakovski, B. Lungwitz, M. I. Gorenstein, and E. L. Bratkovskaya, arXiv:0712.2044 [nucl-th].

[19] C. Alt et al. (NA49 Collaboration), Phys. Rev. C 75, 064904 (2007).

[20] M. M. Aggarwal et al. (WA98 Collaboration), Phys. Rev. C 65, 054912 (2002).

[21] J. T. Mitchell (PHENIX Collaboration), nucl-ex/0510076.
[22] K. Homma (PHENIX Collaboration), in CPOD2006 Proceedings, PoS(CPOD2006)007 (SISSA, Trieste, Italy, 2006).

[23] T. Anticic et al. (NA49 Collaboration), Phys. Rev. C 70, 034902 (2004).

[24] S. Mrowczynski, M. Rybczynski, and Z. Wlodarczyk, Phys. Rev. C 70, 054906 (2004).

[25] L. Cunqueiro, E. G. Ferreiro, F. del Moral, and C. Pajares, Phys. Rev. C 72, 024907 (2005).

[26] K. Grebieszkow et al., arXiv:0707.4608 [nucl-ex].

[27] B. Lungwitz et al. (NA49 Collaboration), arXiv:0709.1646 [nucl-ex].

[28] S. A. Bass et al., Prog. Part. Nucl. Phys. 41, 255 (1998).

[29] I. N. Mishustin, Eur. Phys. J. A 30, 311 (2006).

[30] V. P. Konchakovski, M. I. Gorenstein, and E. L. Bratkovskaya, Phys. Lett. B651, 114 (2007).

[31] H. Heiselberg, Phys. Rep. 351, 161 (2001).

[32] M. Hauer, arXiv:0710.3938 [nucl-th].

[33] S. Afanasiev et al. (NA49 Collaboration), Nucl. Instrum. Methods Phys. Res. A 430, 210 (1999).

[34] C. De Marzo et al., Nucl. Instrum. Methods 217, 405 (1983).

[35] H. Appelshauser et al. (NA49 Collaboration), Eur. Phys. J. A 2 , 383 (1998).

[36] B. Lungwitz, https://edms.cern.ch/document/885236/1.

[37] A. V. Dementev and N. M. Sobolevsky, prepared for 3rd Workshop on Simulating Accelerator Radiation Environments (SARE3), Tsukuba, Japan, 7-9 May 1997 (unpublished).

[38] V. P. Konchakovski, S. Haussler, M. I.. Gorenstein, E. L. Bratkovskaya, M. Bleicher, and H. Stocker, Phys. Rev. C 73, 034902 (2006).

[39] M. Gazdzicki and M. I. Gorenstein, Phys. Lett. B640, 155 (2006).

[40] M. Rybczynski and Z. Wlodarczyk, J. Phys. Conf. Ser. 5, 238 (2005).

[41] K. Werner, Phys. Rep. 232, 87 (1993).

[42] B. Lungwitz et al. (NA49 Collaboration), in CFRNC2006 Proceedings, PoS(CFRNC2006)024 (SISSA, Trieste, Italy, 2006).

[43] B. Lungwitz, AIP Conf. Proc. 892, 400 (2007). 
[44] M. Hauer, V. V. Begun, and M. I. Gorenstein, arXiv:0706.3290 [nucl-th].

[45] M. Bleicher et al., J. Phys. G 25, 1859 (1999), hep-ph/ 9909407.

[46] W. Ehehalt and W. Cassing, Nucl. Phys. A602, 449 (1996).

[47] M. Gazdzicki et al. (NA49 Collaboration), J. Phys. G 30, S701 (2004).
[48] F. Becattini, J. Manninen, and M. Gazdzicki, Phys. Rev. C 73, 044905 (2006).

[49] Y. Hatta and T. Ikeda, Phys. Rev. D 67, 014028 (2003).

[50] M. Gazdzicki et al. (NA49-future Collaboration), in CPOD2006 Proceedings, PoS(CPOD2006)016 (SISSA, Trieste, Italy, 2006).

[51] M. Stephanov (private communication). 